\title{
FINITE SEMIGROUPS THAT ARE MINIMAL FOR NOT BEING MALCEV NILPOTENT
}

\author{
E. JESPERS AND M.H. SHAHZAMANIAN
}

\begin{abstract}
We give a description of finite semigroups $S$ that are minimal for not being Malcev nilpotent, i.e. every proper subsemigroup and every proper Rees factor semigroup is Malcev nilpotent but $S$ is not. For groups this question was considered by Schmidt.
\end{abstract}

\section{INTRODUCTION}

Finite groups $G$ that are minimal for not being nilpotent, i.e. $G$ is not nilpotent but every proper subgroup is nilpotent, have been characterized by Schmidt in [15] (see also [16, Theorem 6.5.7] or [10, Theorem SchmidtRedei-Iwasawa]). For simplicity we call such a group $G$ a Schmidt group. It has the following properties:

(1) $|G|=p^{a} q^{b}$ for some distinct primes $p$ and $q$ and some $a, b>0$.

(2) $G$ has a normal Sylow $p$-subgroup and the Sylow $q$-subgroups are cyclic.

(3) The Frattini subgroups of Sylow subgroups of $G$ are central in $G$.

(4) $G$ is two-generated, i.e. $G=\left\langle g_{1}, g_{2}\right\rangle$ for some $g_{1}, g_{2} \in G$.

It is well known that nilpotent groups can be defined by using semigroup identities (that is without using inverses) and hence there is a natural notion of nilpotent semigroup. This was introduced by Malcev ([11]), and independently by Neuman and Taylor ([12]). For completeness' sake we recall the definition. For elements $x, y, z_{1}, z_{2}, \ldots$ in a semigroup $S$ one recursively defines two sequences

$$
\lambda_{n}=\lambda_{n}\left(x, y, z_{1}, \ldots, z_{n}\right) \quad \text { and } \rho_{n}=\rho_{n}\left(x, y, z_{1}, \ldots, z_{n}\right)
$$

by

$$
\lambda_{0}=x, \quad \rho_{0}=y
$$

and

$$
\lambda_{n+1}=\lambda_{n} z_{n+1} \rho_{n}, \quad \rho_{n+1}=\rho_{n} z_{n+1} \lambda_{n} .
$$

2010 Mathematics Subject Classification. Primary 20F19, 20M07, Secondary: 20F18. Keywords and phrases: semigroup, nilpotent.

The research of the first author is partially supported by Onderzoeksraad of Vrije Universiteit Brussel, Fonds voor Wetenschappelijk Onderzoek (Belgium). A large part of this work was done while the second author was working at Vrije Universiteit Brussel. The second author also gratefully acknowledges support by FCT through the Centro de Matematica da Universidade do Porto (Portugal). 
A semigroup $S$ is said to be nilpotent (in the sense of Malcev [11], denoted $(\mathrm{MN})$ in [6]) if there exists a positive integer $n$ such that

$$
\lambda_{n}\left(a, b, c_{1}, \ldots, c_{n}\right)=\rho_{n}\left(a, b, c_{1}, \ldots, c_{n}\right)
$$

for all $a, b$ in $S$ and $c_{1}, \ldots, c_{n}$ in $S^{1}$ (by $S^{1}$ we denote the smallest monoid containing $S$ ). The smallest such $n$ is called the nilpotency class of $S$. Note that, as in [9], the defining condition to be nilpotent is a bit stronger than the one required by Malcev in [11, who requires elements $w_{i}$ in $S$ only. However the definitions agree on the class of cancellative semigroups. Furthermore, it is shown that a cancellative semigroup $S$ is nilpotent of class $n$ if and only if $S$ has a two-sided group of quotients which is nilpotent of class $n$ (see also [14]). Obviously, other examples of nilpotent semigroups are the power nilpotent semigroups, that is, semigroups $S$ with zero $\theta$ such that $S^{m}=\{\theta\}$ for some $m \geq 1$. In 3 it is shown that a completely 0 -simple semigroup $S$ over a maximal group $G$ is nilpotent if and only if $G$ is nilpotent and $S$ is an inverse semigroup. Of course subsemigroups and Rees factor semigroups of nilpotent semigroups are again nilpotent. The class of 2-nilpotent semigroups has been described in [3]; as for commutative semigroups they have a semilattice decomposition into Archimedean semigroups. For more information on this topic we refer the reader to [3, 5, 6, 7]. In particular, in [7, we describe a class of finite semigroups that are near to being nilpotent, called pseudo nilpotent semigroups. Roughly said, in these semigroups being nilpotent lifts through ideal chains.

In this paper we continue investigating finite semigroups that are close to being nilpotent. Recall that a proper Rees factor semigroup of a semigroup $S$ is a Rees factor semigroup $S / I$ with $I$ an ideal of $S$ of cardinality greater than 1. Obviously, every finite semigroup that is not nilpotent has a subsemigroup that is minimal for not being nilpotent, in the sense that every proper subsemigroup and every Rees factor semigroup is nilpotent. We simply call such a semigroup a minimal non-nilpotent semigroup. The aim of this paper is to describe such semigroups and thus extend Schmidt's investigations to the class of finite semigroups.

The main result (Theorem 4.1) is a classification (of sorts) of minimal non-nilpotent finite semigroups. More specifically, it is shown that such a semigroup is either a Schmidt group or one of four types of semigroups which are not groups. These four types of semigroup are each the union of a completely 0-simple inverse ideal and a 2-generated subsemigroup or a cyclic group. It is also shown that not every semigroup of these four types is minimal non-nilpotent. The proof of the main theorem utilizes the fact that a minimal non-nilpotent semigroup $S$ which is not a group or a semigroup of left or right zeros has a completely 0 -simple inverse ideal $M$ and $S$ acts on the $\mathcal{R}$-classes of $M$. The different types of orbits of this action are analyzed to provide the classification in Theorem 4.1. 
For standard notations and terminology we refer to [2]. A completely 0 -simple finite semigroup $S$ is isomorphic with a regular Rees matrix semigroup $\mathcal{M}^{0}(G, n, m ; P)$, where $G$ is a maximal subgroup of $S, P$ is the $m \times n$ sandwich matrix with entries in $G^{\theta}$ and $n$ and $m$ are positive integers. The nonzero elements of $S$ we denote by $(g ; i, j)$, where $g \in G, 1 \leq i \leq n$ and $1 \leq j \leq m$; the zero element is simply denoted $\theta$. The element of $P$ on the $(i, j)$-position we denote by $p_{i j}$. The set of nonzero elements we denote by $\mathcal{M}(G, n, m ; P)$. If all elements of $P$ are nonzero then this is a semigroup and every completely simple finite semigroup is of this form. If $P=I_{n}$, the identity matrix, then $S$ is an inverse semigroup. By what is mentioned earlier, a completely 0 -simple semigroup $\mathcal{M}^{0}(G, n, m ; P)$ is nilpotent if and only if $n=m, P=I_{n}$ and $G$ is a nilpotent group [3], Lemma 2.1].

The outline of the paper is as follows. In Section 2 we show that a finite minimal non-nilpotent semigroup is either a Schmidt group, or a semigroup with 2 elements of left or right zeros, or $S$ has an ideal $M$ that is a completely 0 -simple inverse semigroup with nilpotent maximal subgroups. In the latter case we prove that $S$ acts on the $\mathcal{R}$-classes of $M$. Next the different types of orbits of this action are analyzed; three cases show up. In Section 3 we deal with each of these cases separately. As a consequence, we obtain in Section 4 a description of finite minimal non-nilpotent semigroups.

\section{Properties of minimal non-Nilpotent Semigroups}

We begin by showing that a finite minimal non-nilpotent semigroup is either a Schmidt group, a non-commutative semigroup with two elements or it has an ideal that is completely 0-simple inverse semigroup with nilpotent maximal subgroups. The starting point of our investigations is the following necessary and sufficient condition for a finite semigroup not to be nilpotent [8].

Lemma 2.1. A finite semigroup $S$ is not nilpotent if and only if there exists a positive integer $m$, distinct elements $x, y \in S$ and elements $w_{1}, w_{2}, \ldots, w_{m} \in$ $S^{1}$ such that $x=\lambda_{m}\left(x, y, w_{1}, w_{2}, \ldots, w_{m}\right), y=\rho_{m}\left(x, y, w_{1}, w_{2}, \ldots, w_{m}\right)$.

Recall that if $S$ is a semigroup with an ideal $I$ such that both $I$ and $S / I$ are nilpotent semigroups then it does not follow in general that $S$ is nilpotent. For counter examples we refer the reader to [3]. However, if $I^{n}=\{\theta\}$ (with $\theta$ the zero element of $S$ ) and $S / I$ is nilpotent then $S$ is a nilpotent semigroup. This easily follows from the previous lemma.

It is easily verified that a finite semigroup of minimal cardinality that is a minimal non-nilpotent semigroup but is not a group is the semigroup of right or left zeros with 2 elements. Obviously, these are bands. For convenience and in order to have a uniform notation for our main result (Theorem 4.1) we will denote such bands respectively as $U_{1}=\{e, f\}$, with ef $=f, f e=e$, and $U_{2}=\left\{e^{\prime}, f^{\prime}\right\}$ with $e^{\prime} f^{\prime}=e^{\prime}, f^{\prime} e^{\prime}=f^{\prime}$. They also can be described as the completely simple semigroups $\mathcal{M}\left(\{e\}, 1,2 ;\left(\begin{array}{l}1 \\ 1\end{array}\right)\right)$ and $\mathcal{M}(\{e\}, 2,1 ;(1,1))$. 
The following result is a first step towards our classification result. It turns out that these are precisely the minimal non-nilpotent finite semigroups that are completely simple.

Lemma 2.2. Let $S$ be a finite semigroup. If $S$ is minimal non-nilpotent then one of the following properties hold:

(1) $S$ is a minimal non-nilpotent group;

(2) $S$ is a semigroup of left or right zeros with 2 elements;

(3) $S$ has a proper ideal which is a completely 0-simple inverse semigroup with nilpotent maximal subgroups, i.e. $S$ has an ideal isomorphic to $\mathcal{M}^{0}\left(G, n, n ; I_{n}\right)$ where $G$ is a nilpotent group and $n \geq 2$.

Proof. Since $S$ is finite, it has a principal series

$$
S=S_{1} \supset S_{2} \supset \cdots \supset S_{h^{\prime}} \supset S_{h^{\prime}+1}=\varnothing .
$$

That is, each $S_{i}$ is an ideal of $S$ and there is no ideal of $S$ strictly between $S_{i}$ and $S_{i+1}$ (for convenience we call the empty set an ideal of $S$ ). Each principal factor $S_{i} / S_{i+1}(1 \leq i \leq m)$ of $S$ is either completely 0 -simple, completely simple or null.

Assume $S$ is minimal non-nilpotent. So, by Lemma 2.1, there exist a positive integer $h$, distinct elements $s_{1}, s_{2} \in S$ and elements $w_{1}, w_{2}, \ldots, w_{h} \in$ $S^{1}$ such that

(1) $s_{1}=\lambda_{h}\left(s_{1}, s_{2}, w_{1}, w_{2}, \ldots, w_{h}\right)$ and $s_{2}=\rho_{h}\left(s_{1}, s_{2}, w_{1}, w_{2}, \ldots, w_{h}\right)$.

Suppose that $s_{1} \in S_{i} \backslash S_{i+1}$. Because $S_{i}$ and $S_{i+1}$ are ideals of $S$, the equalities (11) imply that $s_{2} \in S_{i} \backslash S_{i+1}$ and $w_{1}, w_{2}, \ldots, w_{h} \in S^{1} \backslash S_{i+1}$. Furthermore, one obtains that $S_{i} / S_{i+1}$ is a completely 0 -simple, say $\mathcal{M}^{0}(G, n, m ; P)$, or a completely simple semigroup, say $\mathcal{M}(G, n, m ; P)$. Also, since $S$ is minimal non-nilpotent, $S_{i+1}=\varnothing$ or $S_{i+1}=\{\theta\}$.

If $n+m=2$ then $S_{i} \backslash S_{i+1}$ is a group. We denote by $e$ its identity element. Since $s_{1}, s_{2} \in S_{i} \backslash S_{i+1}$, the sequences $s_{1}=\lambda_{h}\left(s_{1}, s_{2}, w_{1}, w_{2}, \ldots, w_{h}\right)$, $s_{2}=\rho_{h}\left(s_{1}, s_{2}, w_{1}, w_{2}, \ldots, w_{h}\right)$ imply that $\lambda_{j}, \rho_{j} \in S_{i} \backslash S_{i+1}$ for $1 \leq j \leq h$. Now, as $e$ is the identity element of $S_{i} \backslash S_{i+1}, \lambda_{j} w_{j+1} \rho_{i}=\lambda_{j} e w_{j+1} \rho_{i}$ and $\rho_{j} w_{j+1} \lambda_{j}=\rho_{i} e w_{j+1} \lambda_{j}$ for $0 \leq j \leq h-1$. If $S_{i+1}=\{\theta\}$ and $e w_{j+1}=\theta$ for some $0 \leq j \leq h-1$, then $s_{1}=s_{2}=\theta$, in contradiction with $s_{1} \neq s_{2}$. So, $e w_{j+1} \in S_{i} \backslash S_{i+1}$ for $0 \leq j \leq h-1$. Consequently

$$
\begin{gathered}
s_{1}=\lambda_{h}\left(s_{1}, s_{2}, w_{1}, w_{2}, \ldots, w_{h}\right)=\lambda_{h}\left(s_{1}, s_{2}, e w_{1}, e w_{2}, \ldots, e w_{h}\right) \\
\neq s_{2}=\rho_{h}\left(s_{1}, s_{2}, w_{1}, w_{2}, \ldots, w_{h}\right)=\rho_{h}\left(s_{1}, s_{2}, e w_{1}, e w_{2}, \ldots, e w_{h}\right) .
\end{gathered}
$$

From Lemma 2.1 it follows that $S_{i} \backslash S_{i+1}$ is a group that is not nilpotent. Hence, since $S$ is minimal non-nilpotent, $S=S_{i} \backslash S_{i+1}$ and so $S$ is a minimal non-nilpotent group.

Suppose $n+m>2$. If $S_{i} / S_{i+1}$ is not nilpotent then (by the results mentioned in the introduction ([3], Lemma 2.1]) a column or row of $P$ has two nonzero elements. Without loss of generality, we may suppose this is either the first column or the first row. If $p_{i 1}$ and $p_{j 1}$ are nonzero with $i \neq j$ then it 
is easily verified that the subsemigroup $\left\langle\left(p_{i 1}^{-1} ; 1, i\right),\left(p_{j 1}^{-1} ; 1, j\right)\right\rangle$ is isomorphic with the minimal non-nilpotent semigroup $U_{1}$. If, on the other hand, the first row of $P$ contains two nonzero elements, then the semigroup is isomorphic with the minimal non-nilpotent semigroup $U_{2}$. So, $S$ is a semigroup of right or left zeros with two elements.

The remaining case is $n+m>2$ and $S_{i} / S_{i+1}$ is a nilpotent semigroup. Again by [[3], Lemma 2.1], in this case, $S_{i} / S_{i+1}=\mathcal{M}^{0}\left(G, n, n ; I_{n}\right)$ with $G$ a nilpotent group. Since $\left|S_{i+1}\right| \leq 1$, the result follows.

In order to obtain a classification, we thus assume throughout the remainder of this section that $S$ is a finite minimal non-nilpotent semigroup that has a proper ideal $M=\mathcal{M}^{0}\left(G, n, n ; I_{n}\right)$ with $G$ a nilpotent group and $n>1$. To further refine our way towards a classification, we introduce an action of $S$ on the $\mathcal{R}$-classes of $M$, i.e. we define a representation (a semigroup homomorphism)

$$
\Gamma: S \longrightarrow \mathcal{T}_{\{1, \ldots, n\} \cup\{\theta\}},
$$

where $\mathcal{T}$ denotes the full transformation semigroup $\mathcal{T}_{\{1, \ldots, n\} \cup\{\theta\}}$ on the set $\{1, \ldots, n\} \cup\{\theta\}$. The definition is as follows, for $1 \leq i \leq n$ and $s \in S$,

$$
\Gamma(s)(i)= \begin{cases}i^{\prime} & \text { if } s(g ; i, j)=\left(g^{\prime} ; i^{\prime}, j\right) \text { for some } g, g^{\prime} \in G, 1 \leq j \leq n \\ \theta & \text { otherwise }\end{cases}
$$

and

$$
\Gamma(s)(\theta)=\theta .
$$

We call $\Gamma$ a minimal non-nilpotent representation of $S$ and $\Gamma(S)$ a minimal non-nilpotent image of $S$. It is easy to check that $\Gamma$ is well-defined and that it is a semigroup homomorphism.

Also, for every $s \in S$, we define a map

$$
\Psi(s):\{1, \ldots, n\} \cup\{\theta\} \quad \longrightarrow \quad G^{\theta}
$$

as follows

$$
\Psi(s)(i)=g \quad \text { if } \Gamma(s)(i) \neq \theta \text { and } s\left(1_{G} ; i, j\right)=(g ; \Gamma(s)(i), j)
$$

for some $1 \leq j \leq n$, otherwise $\Psi(s)(i)=\theta$. It is straightforward to verify that $\Psi$ is well-defined.

Note that if $\Psi(s)(i)=g$ and $g \in G$ then $s(h ; i, j)=(g h ; \Gamma(s)(i), j)$ for every $h \in G$. Also if $\Psi(s t)(i)=g, \Psi(t)(i)=g^{\prime}$ and $\Psi(s)(\Gamma(t)(i))=g^{\prime \prime}$, then $g=g^{\prime \prime} g^{\prime}$. Hence it follows that

$$
\Psi(s t)=(\Psi(s) \circ \Gamma(t)) \Psi(t) .
$$

We claim that for $s \in S$ the map $\Gamma(s)$ restricted to the domain $S \backslash \Gamma(s)^{-1}(\theta)$ is injective. Indeed, suppose $\Gamma(s)\left(m_{1}\right)=\Gamma(s)\left(m_{2}\right)=m$ for $1 \leq m_{1}, m_{2}, m \leq$ $n$. Then there exist $g, g^{\prime}, h, h^{\prime} \in G, 1 \leq l, l^{\prime} \leq n$ such that $s\left(g ; m_{1}, l\right)=\left(g^{\prime} ; m, l\right)$ and $s\left(h ; m_{2}, l^{\prime}\right)=\left(h^{\prime} ; m, l^{\prime}\right)$. Hence

$$
\left(1_{G} ; m, m\right) s\left(g ; m_{1}, l\right)=\left(g^{\prime} ; m, l\right),\left(1_{G} ; m, m\right) s\left(h ; m_{2}, l^{\prime}\right)=\left(h^{\prime} ; m, l^{\prime}\right)
$$


and thus

$$
\left(1_{G} ; m, m\right) s=\left(x ; m, m_{1}\right)=\left(x^{\prime} ; m, m_{2}\right)
$$

for some $x, x^{\prime} \in G$. It implies that $m_{1}=m_{2}$, as required.

It follows that if $\theta \notin \Gamma(s)(\{1, \ldots, n\})$ then $\Gamma(s)$ induces a permutation on $\{1, \ldots, n\}$ and we may write $\Gamma(s)$ in the disjoint cycle notation (we also write cycles of length one). In the other case, we may write $\Gamma(s)$ as a product of disjoint cycles of the form $\left(i_{1}, i_{2}, \ldots, i_{k}\right)$ or of the form $\left(i_{1}, i_{2}, \ldots, i_{k}, \theta\right)$, where $1 \leq i_{1}, \ldots, i_{k} \leq n$. The notation for the latter cycle means that $\Gamma(s)\left(i_{j}\right)=i_{j+1}$ for $1 \leq j \leq k-1, \Gamma(s)\left(i_{k}\right)=\theta, \Gamma(s)(\theta)=\theta$ and there does not exist $1 \leq r \leq n$ such that $\Gamma(s)(r)=i_{1}$. We also agree that letters $i, j, k$ represent elements of $\{1, \ldots, n\}$, in other words we write explicitly $\theta$ if the zero appears in a cycle. Another agreement we make is that we do not write cycles of the form $(i, \theta)$ in the decomposition of $\Gamma(s)$ if $\Gamma(s)(i)=\theta$ and $\Gamma(s)(j) \neq i$ for every $1 \leq j \leq n$ (this is the reason for writing cycles of length one). If $\Gamma(s)(i)=\theta$ for every $1 \leq i \leq n$, then we simply denote $\Gamma(s)$ as $\theta$.

For convenience, we also introduce the following notation. If the cycle $\varepsilon$ appears in the expression of $\Gamma(s)$ as product of disjoint cycles then we denote this by $\varepsilon \subseteq \Gamma(s)$. If $\Gamma(s)\left(i_{1}\right)=i_{1}^{\prime}, \ldots, \Gamma(s)\left(i_{m}\right)=i_{m}^{\prime}$ then we write

$$
\left[\ldots, i_{1}, i_{1}^{\prime}, \ldots, i_{2}, i_{2}^{\prime}, \cdots, \ldots, i_{m}, i_{m}^{\prime}, \ldots\right] \sqsubseteq \Gamma(s) .
$$

It can be easily verified that if $g \in G$ and $1 \leq n_{1}, n_{2} \leq n$ with $n_{1} \neq n_{2}$ then

$$
\Gamma\left(\left(g ; n_{1}, n_{2}\right)\right)=\left(n_{2}, n_{1}, \theta\right) \text { and } \Gamma\left(\left(g ; n_{1}, n_{1}\right)\right)=\left(n_{1}\right) .
$$

Further, for $s, t \in S, g \in G$ and $1 \leq i \leq n$, if $(\ldots, o, m, k, \ldots) \subseteq \Gamma(s)$ and $\left(m_{1}, \ldots, m_{2}, \theta\right) \subseteq \Gamma(t)$ then

$$
s(g ; m, i)=\left(g^{\prime} ; k, i\right), \quad t\left(g ; m_{2}, i\right)=\theta,
$$

for some $g^{\prime} \in G$. Since $s(g ; o, i)=\left(g^{\prime \prime} ; m, i\right)$ for some $g^{\prime \prime} \in G$ we obtain that

$$
(g ; i, m) s(g ; o, i)=(g ; i, m)\left(g^{\prime \prime} ; m, i\right)=\left(g g^{\prime \prime} ; i, i\right)
$$

and thus $(g ; i, m) s(g ; o, i) \neq \theta$. Hence, there exists $k \in G$ such that

$$
(g ; i, m) s=(k ; i, o) \text {. }
$$

We claim that

$$
\left(g ; i, m_{1}\right) t=\theta
$$

Indeed, suppose this is not the case. Then $\left(g ; i, m_{1}\right) t=\left(g^{\prime} ; i, m_{3}\right)$ for some $g^{\prime} \in G$ and some $m_{3}$. Hence, $\left(g ; i, m_{1}\right) t\left(1_{G} ; m_{3}, m_{3}\right) \neq \theta$ and $t\left(1_{G} ; m_{3}, m_{3}\right) \neq$ $\theta$. So $\Gamma(t)\left(m_{3}\right)=m_{1}$, a contradiction.

In the following lemma we analyze the orbits of this action. Three cases show up.

Lemma 2.3. Let $S$ be a finite minimal non-nilpotent semigroup. Suppose $S$ has proper ideal $M=\mathcal{M}^{0}\left(G, n, n ; I_{n}\right)$ with $G$ a nilpotent group and $n \geq 2$. Then, there exist elements $w_{1}$ and $w_{2}$ of $S \backslash M$ such that one of the following properties holds: 
(i) $(m, l) \subseteq \Gamma\left(w_{1}\right),(m)(l) \subseteq \Gamma\left(w_{2}\right)$,

(ii) $\left(\ldots, m, l, m^{\prime}, \ldots\right) \subseteq \Gamma\left(w_{1}\right),(l)\left(\ldots, m, m^{\prime}, \ldots\right) \subseteq \Gamma\left(w_{2}\right)$,

(iii) $\left[\ldots, k, m, \ldots, l, k^{\prime}, \ldots\right] \subseteq \Gamma\left(w_{1}\right),\left[\ldots, l, m, \ldots, k, k^{\prime}, \ldots\right] \subseteq \Gamma\left(w_{2}\right)$,

for some pairwise distinct numbers $l, m, m^{\prime}, k$ and $k^{\prime}$ between 1 and $n$.

Proof. Because of Lemma 2.1 there exists a positive integer $h$, distinct elements $s_{1}, s_{2} \in S$ and elements $w_{1}, w_{2}, \ldots, w_{h} \in S^{1}$ such that $s_{1}=\lambda_{h}\left(s_{1}, s_{2}, w_{1}\right.$, $\left.w_{2}, \ldots, w_{h}\right), s_{2}=\rho_{h}\left(s_{1}, s_{2}, w_{1}, w_{2}, \ldots, w_{h}\right)$. Note that both $s_{1}$ and $s_{2}$ are nonzero. Because the semigroups $M$ and $S / M$ are nilpotent, $\left\{s_{1}, s_{2}, w_{1}\right.$, $\left.\ldots, w_{h}\right\} \cap M \neq \varnothing$ and $\left\{s_{1}, s_{2}, w_{1}, \ldots, w_{h}\right\} \cap(S \backslash M) \neq \varnothing$. It follows that $s_{1}, s_{2} \in M$ and that there exist $1 \leq n_{1}, n_{2}, n_{3}, n_{4} \leq n$ and $g, g^{\prime} \in G$ such that $s_{1}=\left(g ; n_{1}, n_{2}\right), s_{2}=\left(g^{\prime} ; n_{3}, n_{4}\right)$. Hence

$$
\left[\ldots, n_{3}, n_{2}, \ldots, n_{1}, n_{4}, \ldots\right] \sqsubseteq \Gamma\left(w_{1}\right),\left[\ldots, n_{1}, n_{2}, \ldots, n_{3}, n_{4}, \ldots\right] \sqsubseteq \Gamma\left(w_{2}\right) .
$$

Here we agree that we take $w_{2}=w_{1}$ in case $h=1$.

If $\left(n_{1}, n_{2}\right)=\left(n_{3}, n_{4}\right)$ (for example in the case that $\left.h=1\right)$ then, there exist $k_{i} \in G$ such that $\left(k ; \alpha, n_{2}\right) w_{i}=\left(k k_{i} ; \alpha, n_{1}\right)$ for every $k \in G$ and $\alpha \in\left\{n_{1}, n_{2}\right\}$. Since $\lambda_{i-1}=\left(g_{i-1} ; n_{1}, n_{2}\right), \rho_{i-1}=\left(g_{i-1}^{\prime} ; n_{1}, n_{2}\right)$, for some $g_{i-1}, g_{i-1}^{\prime} \in G$, we get that

$$
\lambda_{i}=\left(g_{i-1} k_{i} g_{i-1}^{\prime} ; n_{1}, n_{2}\right), \rho_{i}=\left(g_{i-1}^{\prime} k_{i} g_{i-1} ; n_{1}, n_{2}\right)
$$

and thus

$$
g=\lambda_{h}\left(g, g^{\prime}, k_{1}, \ldots, k_{h}\right), g^{\prime}=\rho_{h}\left(g, g^{\prime}, k_{1}, \ldots, k_{h}\right) .
$$

Because of Lemma 2.1, this yields a contradiction with $G$ being nilpotent. So we have shown that $\left(n_{1}, n_{2}\right) \neq\left(n_{3}, n_{4}\right)$. In particular, we obtain that $h>1$.

We deal with two mutually exclusive cases.

(Case 1) $n_{1}=n_{2}=l$. Since $\left[\ldots, n_{1}, n_{2}, \ldots, n_{3}, n_{4}, \ldots\right] \subseteq \Gamma\left(w_{2}\right)$, it is impossible that $n_{3}=l, n_{4} \neq l$ or $n_{3} \neq l, n_{4}=l$. As $\left(n_{1}, n_{2}\right) \neq\left(n_{3}, n_{4}\right)$ we thus obtain that $n_{3} \neq l$ and $n_{4} \neq l$. Consequently,

$$
\begin{gathered}
{[\ldots, m, l, \ldots, l, m, \ldots] \subseteq \Gamma\left(w_{1}\right),[\ldots, l, l, \ldots, m, m, \ldots] \subseteq \Gamma\left(w_{2}\right)} \\
\text { or }\left[\ldots, m, l, \ldots, l, m^{\prime}, \ldots\right] \subseteq \Gamma\left(w_{1}\right),\left[\ldots, l, l, \ldots, m, m^{\prime}, \ldots\right] \subseteq \Gamma\left(w_{2}\right)
\end{gathered}
$$

and thus

$$
\begin{gathered}
(m, l) \subseteq \Gamma\left(w_{1}\right),(l)(m) \subseteq \Gamma\left(w_{2}\right) \\
\text { or }\left(\ldots, m, l, m^{\prime}, \ldots\right) \subseteq \Gamma\left(w_{1}\right),(l)\left(\ldots, m, m^{\prime}, \ldots\right) \subseteq \Gamma\left(w_{2}\right)
\end{gathered}
$$

for the pairwise distinct numbers $l, m$ and $m^{\prime}$.

(Case 2) $n_{1} \neq n_{2}$ and $n_{3} \neq n_{4}$ (the latter because otherwise, by symmetry reasons, we are as in Case 1). We obtain five possible cases:

$$
\begin{aligned}
& (1)\left[n_{2}=n_{3}=m\right]: \\
& \quad\left[\ldots, m, m, \ldots, n_{1}, n_{4}, \ldots\right] \sqsubseteq \Gamma\left(w_{1}\right),\left[\ldots, n_{1}, m, \ldots, m, n_{4}, \ldots\right] \sqsubseteq \Gamma\left(w_{2}\right), \\
& (2)\left[n_{2}=n_{4}=m\right]: \\
& \quad\left[\ldots, n_{3}, m, \ldots, n_{1}, m, \ldots\right] \sqsubseteq \Gamma\left(w_{1}\right),\left[\ldots, n_{1}, m, \ldots, n_{3}, m, \ldots\right] \sqsubseteq \Gamma\left(w_{2}\right),
\end{aligned}
$$




$$
\begin{aligned}
& (3)\left[n_{1}=n_{3}=m\right]: \\
& \quad\left[\ldots, m, n_{2}, \ldots, m, n_{4}, \ldots\right] \subseteq \Gamma\left(w_{1}\right),\left[\ldots, m, n_{2}, \ldots, m, n_{4}, \ldots\right] \subseteq \Gamma\left(w_{2}\right), \\
& (4)\left[n_{1}=n_{4}=m\right]: \\
& \quad\left[\ldots, n_{3}, n_{2}, \ldots, m, m, \ldots\right] \subseteq \Gamma\left(w_{1}\right),\left[\ldots, m, n_{2}, \ldots, n_{3}, m, \ldots\right] \subseteq \Gamma\left(w_{2}\right),
\end{aligned}
$$

$(5)$ :

$$
\left[\ldots, k, m, \ldots, l, k^{\prime}, \ldots\right] \sqsubseteq \Gamma\left(w_{1}\right),\left[\ldots, l, m, \ldots, k, k^{\prime}, \ldots\right] \sqsubseteq \Gamma\left(w_{2}\right)
$$

for some pairwise distinct positive integers $l, m, k, k^{\prime} \leq n$.

Cases one and four are as in (ii) of the statement of the lemma. Note that cases two and three are not possible, since $\left(n_{1}, n_{2}\right) \neq\left(n_{3}, n_{4}\right)$ and the restriction of $\Gamma\left(w_{1}\right)$ to $\{1, \ldots, n\} \backslash \Gamma\left(w_{1}\right)^{-1}(\theta)$ is an injective map. Case five is one of the desired options.

Finally, because of (3) we know how the elements of $M$ are written as products of disjoint cycles. Hence it is easily seen that $w_{1}, w_{2} \in(S \backslash M)$.

\section{Three types of SEMigroups}

In this section we deal with each of the cases listed in Lemma 2.3, For the first case we obtain the following description.

Lemma 3.1. Let $S$ be a finite minimal non-nilpotent semigroup. Suppose $M=\mathcal{M}^{0}\left(G, n, n ; I_{n}\right)$ is a proper ideal, with $G$ a nilpotent group and $n \geq 2$. If there exists $u \in S \backslash M$ such that $(m, l) \subseteq \Gamma(u)$, then

$$
S=\mathcal{M}^{0}\left(G, 2,2 ; I_{2}\right) \cup\langle u\rangle
$$

a disjoint union, and

(1) $\langle u\rangle$ a cyclic group of order $2^{k}$,

(2) $u^{2^{k}}=1$ is the identity of $S$,

(3) $\Gamma(u)=(1,2)$ and $\Gamma(1)=(1)(2)$,

(4) $G=\langle\Psi(u)(1), \Psi(u)(2)\rangle$,

(5) $(\Psi(u)(1) \Psi(u)(2))^{2^{k-1}}=1$.

A semigroup $S=\mathcal{M}^{0}\left(G, 2,2 ; I_{2}\right) \cup\langle u\rangle$ that satisfies these five properties is said to be of type $U_{3}$.

Furthermore, $S=\langle(g ; i, j), u\rangle$ for any (nonzero) $(g ; i, j) \in \mathcal{M}^{0}\left(G, 2,2 ; I_{2}\right)$.

Proof. Let $1_{G}$ denote the identity of $G$. Obviously $(m, l) \subseteq \Gamma(u)$ implies that $(m)(l) \subseteq \Gamma\left(u^{2}\right)$. Then because of (3), it is easily seen that

$$
\begin{aligned}
& \Gamma\left(\left(1_{G} ; m, l\right)\right)=\lambda_{2}\left(\Gamma\left(\left(1_{G} ; m, l\right)\right), \Gamma\left(\left(1_{G} ; l, m\right)\right), \Gamma\left(u^{2}\right), \Gamma(u)\right), \\
& \Gamma\left(\left(1_{G} ; l, m\right)\right)=\rho_{2}\left(\Gamma\left(\left(1_{G} ; m, l\right)\right), \Gamma\left(\left(1_{G} ; l, m\right)\right), \Gamma\left(u^{2}\right), \Gamma(u)\right) .
\end{aligned}
$$

Hence the semigroup $\langle u,(g ; m, l),(g ; l, m) \mid g \in G\rangle$ is not nilpotent by Lemma2.1 Since $S$ is minimal non-nilpotent, this implies that

$$
S=\langle u,(g ; m, l),(g ; l, m) \mid g \in G\rangle .
$$


Let $g \in G$. Since $u\left(1_{G} ; m, l\right)=(x ; l, l)$ for some $x \in G$, we obtain that $(g ; m, l) u\left(1_{G} ; m, l\right)=(g x ; m, l) \neq \theta$. Hence,

$$
(g ; m, l) u=(g x ; m, m) .
$$

In particular, $(g ; m, l) u, u(g ; m, l) \in I=\langle(g ; m, l),(g ; l, m) \mid g \in G\rangle$. Note that $I=\mathcal{M}^{0}\left(G, 2,2 ; I_{2}\right)$. Hence $I$ is an ideal in the semigroup $T=\langle u, I\rangle$. Because of (4) and (5) the semigroup $T$ is not nilpotent. Furthermore, for any $u^{\prime} \in\langle u\rangle$ one easily sees that $\Gamma\left(u^{\prime}\right)$ has at least two fixed points or contains a transposition in its disjoint cycle decomposition. Hence, because of (3), $\Gamma\left(u^{\prime}\right) \notin \Gamma(M)$. Therefore, $\langle u\rangle \cap M=\varnothing$.

Consequently, we obtain that $S=\langle u\rangle \cup M$, a disjoint union, $n=2$ and $\Gamma(u)=(m, l)$. It is then clear that in (44) and (5) one may replace $u$ by $u^{k_{1}}$, with $k_{1}$ an odd positive integer. It follows that the subsemigroup $\left\langle u^{k_{1}}, M\right\rangle$ is not nilpotent. Since $S$ is minimal non-nilpotent this implies that $S=$ $\langle u, M\rangle=\left\langle u^{k_{1}}, M\right\rangle$. So $u=u^{r}$ for some positive integer $r \geq 3$. Let $r$ be the smallest such positive integer. Then $u^{r-1}$ is an idempotent and $\langle u\rangle$ is a cyclic group of even order. As $\langle u\rangle=\left\langle u^{k_{1}}\right\rangle$ for any odd positive integer $k_{1}$, we get that $\langle u\rangle$ has order $2^{k}$ for some positive integer $k$.

Without loss of generality we may assume that $m=1, l=2$. As $\left(1_{G} ; 1,1\right) u^{2}$ $=(\Psi(u)(2) \Psi(u)(1) ; 1,1)$ we have

$$
\left(1_{G} ; 1,1\right) u^{2^{k}+1}=\left((\Psi(u)(2) \Psi(u)(1))^{2^{k-1}} \Psi(u)(2) ; 1,2\right) .
$$

Since $\langle u\rangle$ has order $2^{k}, u^{2^{k}+1}=u$ and thus $(\Psi(u)(2) \Psi(u)(1))^{2^{k-1}}=1_{G}$ and $\left(1_{G} ; 1,1\right) u^{2^{k}}=\left(1_{G} ; 1,1\right)$. It follows then easily that $(x ; 1,1) u^{2^{k}}=(x ; 1,1)$ for any $x \in G$. Similarly one obtains that $u^{2^{k}}(x ; 1,1)=(x ; 1,1), u^{2^{k}}(x ; 2,2)=$ $(x ; 2,2) u^{2^{k}}=(x ; 2,2)$ for any $x \in G$. Hence, $u^{2^{k}}$ is the identity of the semigroup $S$.

Let $H=\langle\Psi(u)(1), \Psi(u)(2)\rangle$. From (4) and (5) it easily follows that the subsemigroup

$$
\mathcal{M}^{0}\left(H, 2,2 ; I_{2}\right) \cup\langle u\rangle
$$

is not nilpotent. Then, we obtain that

$$
\mathcal{M}^{0}\left(H, 2,2 ; I_{2}\right) \cup\langle u\rangle=\mathcal{M}^{0}\left(G, 2,2 ; I_{2}\right) \cup\langle u\rangle .
$$

We now show that $G=H$. Suppose the contrary, then there exists $g \in G \backslash H$. Let $\alpha=(g ; 1,1)$. Clearly, $\alpha \notin \mathcal{M}^{0}\left(H, 2,2 ; I_{2}\right)$ and thus $\alpha \in\langle u\rangle$. Since $\Gamma\left(u^{2 k}\right)=(1)(2)$, we get $\Gamma\left(u^{2 k+1}\right)=(1,2)$ for $k>1$ and $\Gamma((g ; 1,1))=(1,1, \theta)$, a contradiction. Thus $G=H$ and $S$ is a semigroup of type $U_{3}$.

Now suppose that $(g ; i, j) \in \mathcal{M}^{0}\left(G, 2,2 ; I_{2}\right)$. If $i=j$ then

$$
\begin{aligned}
\Gamma((g ; i, i)) & =\lambda_{2}\left(\Gamma((g ; i, i)), \Gamma(u(g ; i, i) u), \Gamma(u), \Gamma\left(u^{2}\right)\right), \\
\Gamma(u(g ; i, i) u) & =\rho_{2}\left(\Gamma((g ; i, i)), \Gamma(u(g ; i, i) u), \Gamma(u), \Gamma\left(u^{2}\right)\right) .
\end{aligned}
$$

Hence the semigroup $\langle(g ; i, i), u(g ; i, i) u\rangle$ is not nilpotent by Lemma 2.1 . Since $S$ is minimal non-nilpotent this implies that $S=\langle(g ; i, j), u\rangle$. 
Otherwise if $i \neq j$ we have

$$
\begin{aligned}
& \Gamma((g ; i, j) u)=\lambda_{2}\left(\Gamma((g ; i, j) u), \Gamma(u(g ; i, j)), \Gamma(u), \Gamma\left(u^{2}\right)\right), \\
& \Gamma(u(g ; i, j))=\rho_{2}\left(\Gamma((g ; i, j) u), \Gamma(u(g ; i, j)), \Gamma(u), \Gamma\left(u^{2}\right)\right) .
\end{aligned}
$$

Hence the semigroup $\langle(g ; i, j) u, u(g ; i, j)\rangle$ is not nilpotent by Lemma 2.1. Again since $S$ is minimal non-nilpotent this implies that $S=\langle(g ; i, j), u\rangle$.

Note that not every semigroup of type $U_{3}$ is minimal non-nilpotent. Indeed, let $S=\mathcal{M}^{0}\left(G, 2,2 ; I_{2}\right) \cup\langle u\rangle$, with $\langle u\rangle$ a cyclic group of order $2, u^{2}=1$ is the identity of $S, \Gamma(u)=(1,2), \Gamma(1)=(1)(2), \Psi(u)(1)=g, \Psi(u)(2)=g$ and $G$ a cyclic group $\{1, g\}$. The subsemigroup

$$
\left\{\left(1_{G} ; 1,1\right),\left(1_{G} ; 2,2\right),(g ; 1,2),(g ; 2,1), u, 1, \theta\right\}
$$

is isomorphic with $\mathcal{M}^{0}\left(\{e\}, 2,2 ; I_{2}\right) \cup\langle u\rangle$. As this is a proper semigroup and it is not nilpotent, the semigroup $S$ is of type $U_{3}$ but it is not minimal non-nilpotent.

Semigroups of type $U_{3}$ show up as obstructions in the description, given in [6], of the structure of linear semigroups satisfying certain global and local nilpotence conditions. In particular, it is described when finite semigroups are positively Engel. Recall that a semigroup $S$ is said to be positively Engel, denoted (PE), if for some positive integer $n \geq 2, \lambda_{n}\left(a, b, 1,1, c, c^{2}, \ldots, c^{n-2}\right)=$ $\rho_{n}\left(a, b, 1,1, c, c^{2}, \ldots, c^{n-2}\right)$ for all $a, b$ in $S$ and $c \in S^{1}$. From Corollary 8 in [6] it follows that one of the obstructions for a finite semigroup $S$ to be (PE) is that $S$ has an epimorphic image that has the semigroup $\mathcal{F}_{7}$ of type $U_{3}$ as a subsemigroup, where $\mathcal{F}_{7}=\mathcal{M}^{0}\left(\{e\}, 2,2 ; I_{2}\right) \cup\left\langle u \mid u^{2}=1\right\rangle$.

In order to deal with the second and third cases listed in Lemma 2.3 we prove the following lemma.

Lemma 3.2. Let $S=\mathcal{M}^{0}\left(G, 3,3 ; I_{3}\right) \cup\left\langle w_{1}, w_{2}\right\rangle$ be a semigroup that is the union of the ideal $M=\mathcal{M}^{0}\left(G, 3,3 ; I_{3}\right)$ and the subsemigroup $T=\left\langle w_{1}, w_{2}\right\rangle$. Suppose $\Gamma\left(w_{1}\right)=(2,1,3, \theta)$ and $\Gamma\left(w_{2}\right)=(2,3, \theta)(1)$. Assume $G$ is a nilpotent group, $\theta$ is the zero element of both $M$ and $S$, and suppose $w_{2} w_{1}^{2}=$ $w_{1}^{2} w_{2}=w_{1}^{3}=w_{2} w_{1} w_{2}=\theta$. Then the following properties hold.

(1) $S$ is not nilpotent.

(2) $T$ is nilpotent.

(3) If a subsemigroup $S^{\prime}$ of $S$ is not nilpotent, then $\left\langle w_{1}, w_{2}\right\rangle \subseteq S^{\prime}$.

(4) Every proper Rees factor semigroup of $S$ is nilpotent.

Proof. (1) As

$$
\Gamma\left(\left(1_{G} ; 1,1\right)\right)=\lambda_{2}\left(\Gamma\left(\left(1_{G} ; 1,1\right)\right), \Gamma\left(\left(1_{G} ; 2,3\right)\right), \Gamma\left(w_{1}\right), \Gamma\left(w_{2}\right)\right)
$$

and

$$
\Gamma\left(\left(1_{G} ; 2,3\right)\right)=\rho_{2}\left(\Gamma\left(\left(1_{G} ; 1,1\right)\right), \Gamma\left(\left(1_{G} ; 2,3\right)\right), \Gamma\left(w_{1}\right), \Gamma\left(w_{2}\right)\right)
$$

we get from Lemma 2.1 that $S$ is not nilpotent. 
(2) Clearly $I=T \backslash\left\langle w_{2}\right\rangle$ is an ideal of $T$ and $I^{3}=\{\theta\}$. Obviously $T / I$ is commutative and thus nilpotent. Hence, $T$ is nilpotent.

(3) Assume $S^{\prime}$ is a subsemigroup of $S$ that is not nilpotent. Again by Lemma 2.1. there exists a positive integer $p$, distinct elements $t, t^{\prime} \in S^{\prime}$ and $t_{1}, t_{2}, \ldots, t_{p} \in S^{\prime 1}$ such that $t=\lambda_{p}\left(t, t^{\prime}, t_{1}, t_{2}, \ldots, t_{p}\right), t^{\prime}=\rho_{p}\left(t, t^{\prime}, t_{1}, t_{2}, \ldots, t_{p}\right)$. Since $T$ is nilpotent,

$$
\left\{t, t^{\prime}, t_{1}, t_{2}, \ldots, t_{p}\right\} \cap \mathcal{M}^{0}\left(G, 3,3 ; I_{3}\right) \neq \varnothing
$$

and since $\mathcal{M}^{0}\left(G, 3,3 ; I_{3}\right)$ is an ideal of $S, t$ and $t^{\prime}$ are in $\mathcal{M}^{0}\left(G, 3,3 ; I_{3}\right)$. Since $S^{\prime}$ is not nilpotent and $\mathcal{M}^{0}\left(G, 3,3 ; I_{3}\right)$ is nilpotent, we obtain that at least one of the elements $t_{1}, \ldots, t_{p}$ is in $T$. Now, if necessary, replacing $t$ by $\lambda_{i-1}\left(t, t^{\prime}, t_{1}, t_{2}, \ldots, t_{i-1}\right)$ and $t^{\prime}$ by $\rho_{i-1}\left(t, t^{\prime}, t_{1}, t_{2}, \ldots, t_{i-1}\right)$, we may assume that $t_{1} \in T$.

Write $t=\left(g_{1} ; n_{1}, n_{2}\right)$ and $t^{\prime}=\left(g_{2} ; n_{3}, n_{4}\right)$, for some $1 \leq n_{1}, n_{2}, n_{3}, n_{4} \leq 3$ and $g_{1}, g_{2} \in G$.

Consider the following subsets of $T: A=\left\{w_{1}\right\}, B=\left\{w_{2}\right\}, C=\left\{w_{2}^{n} \mid n \epsilon\right.$ $\mathbb{N}, n>1\}, D=\left\{w_{1} w_{2}^{n} w_{1} \mid n \in \mathbb{N}\right\}, E=\left\{w_{1} w_{2}^{n} \mid n \in \mathbb{N}, n>0\right\}, F=\left\{w_{2}^{n} w_{1} \mid\right.$ $n \in \mathbb{N}, n>0\}$ and $Z=\left\{T^{1} w_{1}^{2} T^{1}, T^{1} w_{2} x_{1} w_{2} T^{1}\right\} \backslash\left\{w_{1}^{2}\right\}$. By determining the images of these sets under the mapping $\Gamma$ one sees that these sets form a partition of $T$. Since $w_{2} w_{1}^{2}=w_{1}^{2} w_{2}=w_{1}^{3}=w_{2} w_{1} w_{2}=\theta$ we have that $Z=\{\theta\}$. Hence $t_{1} \notin Z$.

If $t_{1} \in C$ then $n_{1}=n_{2}=n_{3}=n_{4}=1$ (because, for every $a \in C$ we have $\Gamma(a)=(1))$ and thus $g_{1}=\lambda_{p}\left(g_{1}, g_{2}, x_{1}, \ldots, x_{p}\right)$ and $g_{2}=\rho_{p}\left(g_{1}, g_{2}, x_{1}, \ldots, x_{p}\right)$ for some $x_{1}, \ldots, x_{p} \in G$, in contradiction with $G$ being nilpotent. If $t_{1} \in$ $D$ then $n_{1}=n_{3}=2$ and $n_{2}=n_{4}=3$ (because for every $a \in D$ we have $\Gamma(a)=(2,3, \theta))$; this again yields a contradiction with $G$ being nilpotent. Similarly $t_{1} \notin E, F$. Now suppose that $t_{1} \in A$, i.e. $t_{1}=w_{1}$. Since $\Gamma\left(w_{1}\right)=$ $(2,1,3, \theta), t=\lambda_{p}\left(t, t^{\prime}, t_{1}, t_{2}, \ldots, t_{p}\right) \neq \theta$ and $t^{\prime}=\rho_{p}\left(t, t^{\prime}, t_{1}, \ldots, t_{p}\right) \neq \theta$ we get that $\left\{n_{1}, n_{3}\right\} \subseteq\{1,2\}$. As $t w_{1} t^{\prime} \neq \theta$ and $t^{\prime} w_{1} t \neq \theta$ we obtain that $n_{2}=$ $\Gamma\left(w_{1}\right)\left(n_{3}\right)$ and $n_{4}=\Gamma\left(w_{1}\right)\left(n_{1}\right)$. Hence, if $n_{1}=n_{3}$, then $n_{2}=n_{4}$, again yielding a contradiction with $G$ being nilpotent. So, $n_{1} \neq n_{3}$. If $n_{1}=1$ then $n_{3}=2, n_{4}=3, n_{2}=1$ and thus then $\left\{\left(n_{1}, n_{2}\right),\left(n_{3}, n_{4}\right)\right\}=\{(1,1),(2,3)\}$. Similarly, we also get the latter if $n_{1}=2$. Note that in this case $p>1$. It then can be easily verified that $t_{2}=w_{2}$ and thus $T \subseteq S^{\prime}$, as desired. Similarly if $t_{1}=w_{2}$, then $T \subseteq S^{\prime}$.

(4) Since $M$ is a completely 0 -simple semigroup and because $T$ is nilpotent, it is clear that every proper Rees factor of $S$ is nilpotent.

We now are in a position to obtain a description of finite minimal nonnilpotent semigroups that are not of type $U_{3}$ and that have a proper ideal that is a completely 0 -simple inverse semigroup.

Lemma 3.3. Let $S$ be a finite minimal non-nilpotent semigroup with a proper ideal $M=\mathcal{M}^{0}\left(G, n, n ; I_{n}\right), G$ a nilpotent group and $n \geq 2$. Suppose $S$ is not of type $U_{3}$, i.e. for $x \in S \backslash M$ there do not exist distinct numbers $l_{1}$ 
and $l_{2}$ between 1 and $n$ such that $\left(l_{1}, l_{2}\right) \subseteq \Gamma(x)$. Then $S$ is a semigroup of one of the following two types.

(1) $S=\mathcal{M}^{0}\left(G, 3,3 ; I_{3}\right) \cup\left\langle x_{1}, x_{2}\right\rangle$, with $\mathcal{M}^{0}\left(G, 3,3 ; I_{3}\right)$ a proper ideal of $S$, $\Gamma\left(x_{1}\right)=(2,1,3, \theta), \Gamma\left(x_{2}\right)=(2,3, \theta)(1), x_{2} x_{1}^{2}=x_{1}^{2} x_{2}=x_{1}^{3}=x_{2} x_{1} x_{2}=$ $\theta$ (the zero element of $S$ ),

$$
G=\left\langle\Psi\left(x_{1}\right)(1), \Psi\left(x_{1}\right)(2), \Psi\left(x_{2}\right)(1), \Psi\left(x_{2}\right)(2)\right\rangle .
$$

Such a semigroup is said to be of type $U_{4}$.

(2) $S=\mathcal{M}^{0}\left(G, n, n ; I_{n}\right) \cup\left\langle v_{1}, v_{2}\right\rangle$, with $\mathcal{M}^{0}\left(G, n, n ; I_{n}\right)$ a proper ideal of $S$

$$
\left[\ldots, k_{1}, k_{2}, \ldots, k_{3}, k_{4}, \ldots\right] \sqsubseteq \Gamma\left(v_{1}\right),\left[\ldots, k_{1}, k_{4}, \ldots, k_{3}, k_{2}, \ldots\right] \sqsubseteq \Gamma\left(v_{2}\right)
$$

for pairwise distinct numbers $k_{1}, k_{2}, k_{3}$ and $k_{4}$ between 1 and $n, G=$ $\left\langle\Psi\left(v_{1}\right)(1), \ldots, \Psi\left(v_{1}\right)(n), \Psi\left(v_{2}\right)(1), \ldots, \Psi\left(v_{2}\right)(n), \theta\right\rangle \backslash\{\theta\}$ and there do not exist pairwise distinct numbers $o_{1}, o_{2}$ and $o_{3}$ between 1 and $n$ such that $\left(o_{2}, o_{1}, o_{3}, \theta\right) \subseteq \Gamma(y),\left(o_{2}, o_{3}, \theta\right)\left(o_{1}\right) \subseteq \Gamma(z)$ for some $y, z \in$ $\left\langle v_{1}, v_{2}\right\rangle$. Such a semigroup is said to be of type $U_{5}$.

Furthermore, if $S$ of type $U_{4}$ then $S=\left\langle(g ; 1,1),\left(g^{\prime} ; 2,3\right), x_{1}, x_{2}\right\rangle$ and if $S$ is of type $U_{5}$ then $S=\left\langle\left(g ; k_{1}, k_{4}\right),\left(g^{\prime} ; k_{3}, k_{2}\right), v_{1}, v_{2}\right\rangle$, for every $g, g^{\prime} \in G$.

Proof. For clarity we give a brief outline of the structure of the proof. By assumption $S$ is not of type $U_{3}$ and hence it follows from Lemma 2.3 that we have two cases to deal with and this is done in three parts. In part (1) we deal with a special case of part (ii) of Lemma 2.3. In the remainder of the proof we then assume that we are not in this special case. In part (2) we deal with all cases occurring in part (iii) of Lemma 2.3 as well as in part (ii) of Lemma 2.3, the latter provided some extra condition is satisfied. Finally in part (3), we show that if this extra assumption is not satisfied then $S$ has to be of type $U_{5}$.

Part (1). We begin the proof with handling a special case stated in part (ii) of Lemma 2.3. Suppose that there exist elements $x_{1}, x_{2} \in S$ such that

$$
\left(o_{2}, o_{1}, o_{3}, \theta\right) \subseteq \Gamma\left(x_{1}\right) \text { and }\left(o_{2}, o_{3}, \theta\right)\left(o_{1}\right) \subseteq \Gamma\left(x_{2}\right) \text {, }
$$

with $o_{1}, o_{2}, o_{3}$ positive integers between 1 and $n$. It is then readily verified that $\mathcal{M}^{0}\left(G,\left\{o_{1}, o_{2}, o_{3}\right\},\left\{o_{1}, o_{2}, o_{3}\right\} ; I_{\left\{o_{1}, o_{2}, o_{3}\right\}}\right)$ is an ideal in the subsemigroup $\mathcal{M}^{0}\left(G,\left\{o_{1}, o_{2}, o_{3}\right\},\left\{o_{1}, o_{2}, o_{3}\right\} ; I_{\left\{o_{1}, o_{2}, o_{3}\right\}}\right) \cup\left\langle x_{1}, x_{2}\right\rangle$ of $S$. Because $(11) \Gamma\left(\left(1_{G} ; o_{1}, o_{1}\right)\right)=\lambda_{2}\left(\Gamma\left(\left(1_{G} ; o_{1}, o_{1}\right)\right), \Gamma\left(\left(1_{G} ; o_{2}, o_{3}\right)\right), \Gamma\left(x_{1}\right), \Gamma\left(x_{2}\right)\right)$, $(12) \Gamma\left(\left(1_{G} ; o_{2}, o_{3}\right)\right)=\rho_{2}\left(\Gamma\left(\left(1_{G} ; o_{1}, o_{1}\right)\right), \Gamma\left(\left(1_{G} ; o_{2}, o_{3}\right)\right), \Gamma\left(x_{1}\right), \Gamma\left(x_{2}\right)\right)$

we get that the semigroup $\mathcal{M}^{0}\left(G,\left\{o_{1}, o_{2}, o_{3}\right\},\left\{o_{1}, o_{2}, o_{3}\right\} ; I_{\left\{o_{1}, o_{2}, o_{3}\right\}}\right) \cup\left\langle x_{1}, x_{2}\right\rangle$ is not nilpotent and thus, as $S$ is minimal non-nilpotent, $S=\mathcal{M}^{0}\left(G, 3,3 ; I_{3}\right) \cup$ $\left\langle x_{1}, x_{2}\right\rangle$.

We now prove that $S$ is a semigroup of type $U_{4}$. We do so by showing that $x_{1}$ and $x_{2}$ satisfy conditions listed in part (1) of the statement of the lemma. Let $T=\left\langle x_{1}, x_{2}\right\rangle$. Consider the following subsets of $T: A=\left\{x_{1}\right\}, B=\left\{x_{2}\right\}$, $C=\left\{x_{2}^{n} \mid n \in \mathbb{N}, n>1\right\}, D=\left\{x_{1} x_{2}^{n} x_{1} \mid n \in \mathbb{N}\right\}, E=\left\{x_{1} x_{2}^{n} \mid n \in \mathbb{N}, n>0\right\}, F=$ 
$\left\{x_{2}^{n} x_{1} \mid n \in \mathbb{N}, n>0\right\}$ and $Z=\left\{T^{1} x_{1}^{2} T^{1}, T^{1} x_{2} x_{1} x_{2} T^{1}\right\} \backslash\left\{x_{1}^{2}\right\}$. By determining the images of these sets under the mapping $\Gamma$ one sees that these sets form a partition of $T$. Since $S^{\prime}=\left\{T^{1} x_{1}^{2} T^{1}, T^{1} x_{2} x_{1} x_{2} T^{1}\right\} \backslash\left\{x_{1}^{2}\right\} \cup\{\theta\}$ is an ideal of $S$, it easily follows from (111) and (12) that $S / S^{\prime}$ is non-nilpotent. Hence, $S^{\prime}=\{\theta\}$ and thus $Z=\{\theta\}$. So, $x_{1}^{3}=x_{1}^{2} x_{2}=x_{2} x_{1}^{2}=x_{2} x_{1} x_{2}=\theta$, as desired. From Lemma $3.2(2)$ we know that the subsemigroup $T=\left\langle x_{1}, x_{2}\right\rangle$ is nilpotent.

Let $H=\left\langle\Psi\left(x_{1}\right)\left(o_{1}\right), \Psi\left(x_{1}\right)\left(o_{2}\right), \Psi\left(x_{2}\right)\left(o_{1}\right), \Psi\left(x_{2}\right)\left(o_{2}\right)\right\rangle$. For simplicity, and without loss of generality, we may assume that $o_{1}=1, o_{2}=2$ and $o_{3}=3$. From (11) and (12) it can be easily verified that the subsemigroup

$$
\mathcal{M}^{0}\left(\left\langle\Psi\left(x_{1}\right)(1), \Psi\left(x_{1}\right)(2), \Psi\left(x_{2}\right)(1), \Psi\left(x_{2}\right)(2)\right\rangle, 3,3 ; I_{3}\right) \cup\left\langle x_{1}, x_{2}\right\rangle
$$

is not nilpotent. Hence

$$
\mathcal{M}^{0}\left(H, 3,3 ; I_{3}\right) \cup\left\langle x_{1}, x_{2}\right\rangle=\mathcal{M}^{0}\left(G, 3,3 ; I_{3}\right) \cup\left\langle x_{1}, x_{2}\right\rangle .
$$

We now show that $G=H$. Suppose the contrary and let $g \in G \backslash H$. Let $\alpha=(g ; 1,1)$. Clearly, $\alpha \notin \mathcal{M}^{0}\left(H, 3,3 ; I_{3}\right)$ and thus $\alpha \in\left\langle x_{1}, x_{2}\right\rangle$. Since $(g ; 1,1)\left(1_{G} ; 1,1\right) \neq \theta$, we get that $\Gamma(\alpha)(1), \Psi(\alpha)(1) \neq \theta$ and $(g ; 1,1)=$ $(g ; 1,1)\left(1_{G} ; 1,1\right)=\alpha\left(1_{G} ; 1,1\right)=(\Psi(\alpha)(1) ; \Gamma(\alpha)(1), 1)$ and thus $g=\Psi(\alpha)(1)$. This contradicts with $g \notin H$. So, indeed, $G=H$. Hence we have shown that indeed $S$ is a semigroup of type $U_{4}$. To prove the last part of the statement of the lemma for this semigroup, let $g, g^{\prime} \in G$. Since

$$
\Gamma((g ; 1,1))=\lambda_{2}\left(\Gamma((g ; 1,1)), \Gamma\left(\left(g^{\prime} ; 2,3\right)\right), \Gamma\left(x_{1}\right), \Gamma\left(x_{2}\right)\right)
$$

and

$$
\Gamma\left(\left(g^{\prime} ; 2,3\right)\right)=\rho_{2}\left(\Gamma((g ; 1,1)), \Gamma\left(\left(g^{\prime} ; 2,3\right)\right), \Gamma\left(x_{1}\right), \Gamma\left(x_{2}\right)\right),
$$

we get that the subsemigroup $\left\langle(g ; 1,1),\left(g^{\prime} ; 2,3\right), x_{1}, x_{2}\right\rangle$ is not nilpotent. Since $S$ is minimal non-nilpotent it follows that $S=\left\langle(g ; 1,1),\left(g^{\prime} ; 2,3\right), x_{1}\right.$, $\left.x_{2}\right\rangle$.

Part (2). In the remainder of the proof we assume that there do not exist pairwise distinct numbers $o_{1}, o_{2}$ and $o_{3}$ between 1 and $n$ such that $\left(o_{2}, o_{1}, o_{3}, \theta\right) \subseteq \Gamma(y),\left(o_{2}, o_{3}, \theta\right)\left(o_{1}\right) \subseteq \Gamma(z)$ for some $y, z \in S$. Because, by assumption $S$ is not of type $U_{3}$, it follows from Lemma 2.3 that $S \backslash M$ contains elements $w_{1}$ and $w_{2}$ such that

$$
\left(\ldots, m, l, m^{\prime}, \ldots\right) \subseteq \Gamma\left(w_{1}\right),(l)\left(\ldots, m, m^{\prime}, \ldots\right) \subseteq \Gamma\left(w_{2}\right),
$$

or it contains elements $v_{1}$ and $v_{2}$ such that

$$
\left[\ldots, k, m, \ldots, l, k^{\prime}, \ldots\right] \subseteq \Gamma\left(v_{1}\right),\left[\ldots, l, m, \ldots, k, k^{\prime}, \ldots\right] \subseteq \Gamma\left(v_{2}\right),
$$

for some pairwise distinct numbers $l, m, m^{\prime}, k$ and $k^{\prime}$ between 1 and $n$. In the former case, without loss of generality, we may assume that $m=1, l=3$ and $m^{\prime}=2$.

Assume the former case holds, i.e.

$$
(\ldots, 1,3,2, \ldots) \subseteq \Gamma\left(w_{1}\right) \text { and }(3)(\ldots, 1,2, \ldots) \subseteq \Gamma\left(w_{2}\right),
$$

and also suppose that

$$
\Gamma\left(w_{1}\right)(2)=r \neq \theta
$$


Then, $[\ldots, 1,2, \ldots, 3, r, \ldots] \sqsubseteq \Gamma\left(w_{1}^{2}\right),[\ldots, 1, r, \ldots, 3,2, \ldots] \sqsubseteq \Gamma\left(w_{1} w_{2}\right)$. Hence, $v_{1}=w_{1}^{2}$ and $v_{2}=w_{1} w_{2}$ are elements as in the latter case. For such elements $\Gamma\left(v_{1}\right)=[\ldots, 1,2, \ldots, 3, r, \ldots]$ and $\Gamma\left(v_{2}\right)=[\ldots, 1, r, \ldots, 3,2, \ldots]$ we get that

$$
\Gamma\left(\left(1_{G} ; 1, r\right)\right)=\lambda_{2}\left(\Gamma\left(\left(1_{G} ; 1, r\right)\right), \Gamma\left(\left(1_{G} ; 3,2\right)\right), \Gamma\left(v_{1}\right), \Gamma\left(v_{2}\right)\right)
$$

and

$$
\Gamma\left(\left(1_{G} ; 3,2\right)\right)=\rho_{2}\left(\Gamma\left(\left(1_{G} ; 1, r\right)\right), \Gamma\left(\left(1_{G} ; 3,2\right)\right), \Gamma\left(v_{1}\right), \Gamma\left(v_{2}\right)\right) .
$$

It follows that the subsemigroup $\mathcal{M}^{0}\left(G, n, n ; I_{n}\right) \cup\left\langle v_{1}, v_{2}\right\rangle$ is not nilpotent. So, because $S$ is minimal non-nilpotent, $S=\mathcal{M}^{0}\left(G, n, n ; I_{n}\right) \cup\left\langle v_{1}, v_{2}\right\rangle$. Furthermore, it is easily verified that

$$
\Gamma((g ; 1, r))=\lambda_{2}\left(\Gamma((g ; 1, r)), \Gamma\left(\left(g^{\prime} ; 3,2\right)\right), \Gamma\left(v_{1}\right), \Gamma\left(v_{2}\right)\right)
$$

and

$$
\Gamma\left(\left(g^{\prime} ; 3,2\right)\right)=\rho_{2}\left(\Gamma((g ; 1, r)), \Gamma\left(\left(g^{\prime} ; 3,2\right)\right), \Gamma\left(v_{1}\right), \Gamma\left(v_{2}\right)\right) .
$$

Hence, for any $g, g^{\prime} \in G$, the subsemigroup $\left\langle(g ; 1, r),\left(g^{\prime} ; 3,2\right), v_{1}, v_{2}\right\rangle$ is not nilpotent. Therefore, $S=\left\langle(g ; 1, r),\left(g^{\prime} ; 3,2\right), v_{1}, v_{2}\right\rangle$ for every $g, g^{\prime} \in G$. We now show that such a semigroup $S$ is of type $U_{5}$. Let

$$
H_{1}=\left\langle\Psi\left(v_{1}\right)(1), \ldots, \Psi\left(v_{1}\right)(n), \Psi\left(v_{2}\right)(1), \ldots, \Psi\left(v_{2}\right)(n), \theta\right\rangle .
$$

Note that $H=H_{1} \backslash\{\theta\}$ is a subgroup of the maximal subgroup $G$ defining $M$. Since

$$
\begin{aligned}
& \Gamma\left(\left(1_{G} ; 3,2\right)\right)=\lambda_{2}\left(\Gamma\left(\left(1_{G} ; 3,2\right)\right), \Gamma\left(\left(1_{G} ; 1, r\right)\right), \Gamma\left(v_{1}\right), \Gamma\left(v_{2}\right)\right), \\
& \Gamma\left(\left(1_{G} ; 1, r\right)\right)=\rho_{2}\left(\Gamma\left(\left(1_{G} ; 3,2\right)\right), \Gamma\left(\left(1_{G} ; 1, r\right)\right), \Gamma\left(v_{1}\right), \Gamma\left(v_{2}\right)\right)
\end{aligned}
$$

we get that $\mathcal{M}^{0}\left(H, n, n ; I_{n}\right) \cup\left\langle v_{1}, v_{2}\right\rangle$ is not nilpotent. Because, by assumption, $S$ is minimal non-nilpotent, we obtain that

$$
\mathcal{M}^{0}\left(H, n, n ; I_{n}\right) \cup\left\langle v_{1}, v_{2}\right\rangle=\mathcal{M}^{0}\left(G, n, n ; I_{n}\right) \cup\left\langle v_{1}, v_{2}\right\rangle .
$$

We now show that $G=H$. Suppose the contrary and let $g \in G \backslash H$. Let $\alpha=(g ; 1,1)$. Clearly, $\alpha \notin \mathcal{M}^{0}\left(H, n, n ; I_{n}\right)$ and thus $\alpha \in\left\langle v_{1}, v_{2}\right\rangle$. Since $(g ; 1,1)\left(1_{G} ; 1,1\right) \neq \theta$ we get that $\Gamma(\alpha)(1) \neq \theta, \Psi(\alpha)(1) \neq \theta$ and

$$
(g ; 1,1)=(g ; 1,1)\left(1_{G} ; 1,1\right)=\alpha\left(1_{G} ; 1,1\right)=(\Psi(\alpha)(1) ; \Gamma(\alpha)(1), 1)
$$

Thus $g=\Psi(\alpha)(1)$. This contradicts with $g \notin H$. So, indeed, $G=H$.

Part (3). We are left to deal with the case that $S \backslash M$ contains elements $w_{1}$ and $w_{2}$ such that

$$
(\ldots, 1,3,2, \ldots) \subseteq \Gamma\left(w_{1}\right),(3)(\ldots, 1,2, \ldots) \subseteq \Gamma\left(w_{2}\right),
$$

and

$$
\Gamma\left(w_{1}\right)(2)=\theta \text {. }
$$

We show that if $S$ is not of type $U_{5}$ then this can not occur. First notice that we also may assume that there does not exist a positive integer $r^{\prime}$, with $1 \leq r^{\prime} \leq n$, such that $\Gamma\left(w_{1}\right)\left(r^{\prime}\right)=1$. Indeed, suppose the contrary and let $r^{\prime}$ be such a positive integer. Then $\left[\ldots, 1,2, \ldots, r^{\prime}, 3, \ldots\right] \sqsubseteq$ 
$\Gamma\left(w_{1}^{2}\right),\left[\ldots, 1,3, \ldots, r^{\prime}, 2, \ldots\right] \sqsubseteq \Gamma\left(w_{2} w_{1}\right)$ and thus $S$ is a semigroup of type $U_{5}$. This proves the claim. Thus

$$
(1,3,2, \theta) \subseteq \Gamma\left(w_{1}\right) .
$$

Next we claim that the cycle $(\ldots, 1,2, \ldots)$ in $\Gamma\left(w_{2}\right)$ ends in $\theta$. Indeed, assume the contrary. That is, this cycle ends in a positive integer. Let $n_{2}$ denote the length of this cycle. Then, $(1,3) \subseteq \Gamma\left(w_{2}^{n_{2}-1} w_{1}\right),(1)(3) \subseteq$ $\Gamma\left(w_{2}^{n_{2}}\right)$. However, this is excluded by assumption. This proves the claim and thus there exist positive integers $k, k^{\prime}$ and $n^{\prime}$ such that $\Gamma\left(w_{2}\right)(k)=k^{\prime} \neq \theta$, $\Gamma\left(w_{2}\right)\left(k^{\prime}\right)=\theta$ and $\Gamma\left(w_{2}^{n^{\prime}}\right)(1)=k^{\prime}$. So

$$
\text { (3) }\left(\ldots, 1,2, \ldots, k, k^{\prime}, \theta\right) \subseteq \Gamma\left(w_{2}\right) \text {. }
$$

If $\Gamma\left(w_{2}^{n^{\prime}-1} w_{1}\right)\left(k^{\prime}\right)=k^{\prime \prime} \neq \theta$ then $\left[\ldots, 1, k^{\prime}, \ldots, 3, k^{\prime \prime}, \ldots\right] \sqsubseteq \Gamma\left(\left(w_{2}^{n^{\prime}-1} w_{1}\right)^{2}\right)$, $\left[\ldots, 1, k^{\prime \prime}, \ldots, 3, k^{\prime}, \ldots\right] \sqsubseteq \Gamma\left(w_{2}^{n^{\prime}-1} w_{1} w_{2}^{n^{\prime}}\right)$. Since $\Gamma\left(w_{2}\right)(3)=3, \Gamma\left(w_{2}\right)(1)=2$ and $\Gamma\left(w_{2}\right)\left(k^{\prime}\right)=\theta$, it is clear that $1, k^{\prime}$ and 3 are pairwise distinct. As $\Gamma\left(w_{2}^{n^{\prime}-1} w_{1}\right)\left(k^{\prime}\right)=k^{\prime \prime} \neq \theta$, we get that $\Gamma\left(w_{1}\right)\left(k^{\prime}\right)=\alpha \neq \theta$. As $(1,3,2, \theta) \subseteq$ $\Gamma\left(w_{1}\right)$ and because $1, k^{\prime}, 3$ are pairwise distinct positive integers, we get that $\alpha \notin\{1,2,3\}$. We claim that $1, k^{\prime}, 3$ and $k^{\prime \prime}$ are pairwise distinct and thus that $S$ is a semigroup of type $U_{5}$, yielding a contradiction. Indeed, for otherwise, $k^{\prime \prime}=3$ or $k^{\prime \prime}=k^{\prime}$ or $k^{\prime \prime}=1$. The former is excluded as it implies that $\alpha=3$. If $k^{\prime \prime}=k^{\prime}$, then $\alpha=2$, a contradiction. If $k^{\prime \prime}=1$ then $(\alpha, 3) \subseteq$ $\Gamma\left(w_{1} w_{2}^{n^{\prime}-1} w_{1} w_{2}^{2 n^{\prime}-1}\right)$ and thus $S$ is of type $U_{3}$, again a contradiction. This proves the claim. Finally, if $\Gamma\left(w_{2}^{n^{\prime}-1} w_{1}\right)\left(k^{\prime}\right)=\theta$, we get that $\left(1,3, k^{\prime}, \theta\right) \subseteq$ $\Gamma\left(w_{2}^{n^{\prime}-1} w_{1}\right)$. Clearly, $(3)\left(1, k^{\prime}, \theta\right) \subseteq \Gamma\left(w_{2}^{n^{\prime}}\right)$. However, this contradicts with our assumptions. This final contradiction shows that indeed this considered case does not occur.

\section{MAin Result And EXAmples}

We are now in a position to state and prove the main result.

Theorem 4.1. Let $S$ be a finite minimal non-nilpotent semigroup. Then $S$ is either a Schmidt group or a semigroup of type $U_{1}, U_{2}, U_{3}, U_{4}$ or $U_{5}$. In particular, the semigroups $S$ of type $U_{m}$, with $3 \leq m \leq 5$, are generated by four elements, they have a two-generated subsemigroup $T$ and an ideal $M=\mathcal{M}^{0}\left(G, n, n ; I_{n}\right)$ (with $G$ a nilpotent group) such that

$$
S=M \cup T,
$$

and there exists a representation

$$
\Gamma: S \longrightarrow \mathcal{T}_{\{1, \ldots, n\} \cup\{\theta\}},
$$

such that, for all $s \in S$,

(1) $\Gamma(s)(\theta)=\theta$,

(2) $\Gamma(s)$ is injective when restricted to $\{1, \ldots, n\} \backslash \Gamma(s)^{-1}(\theta)$,

(3) $\left|\Gamma^{-1}(\theta)\right|=1$, 
(4) if $T$ has a zero element, say $\theta_{T}$, then $\theta_{T}=\theta$ (the zero of $S$ ).

Furthermore, $\Gamma(S)$ also is a minimal non-nilpotent semigroup.

Proof. All parts, except the items (3) and (4), follow at once from Lemma2.2, Lemma 2.3. Lemma 3.1 and Lemma 3.3. Part (3) follows from the fact that $\Gamma^{-1}(\theta)$ is an ideal of $S$ and $S / \Gamma^{-1}(\theta)$ is not nilpotent.

To prove part (4), assume $T$ has a zero element, say $\theta_{T}$. We prove by contradiction that $\theta_{T}=\theta$. So suppose that $\theta_{T} \neq \theta$. Then, by part (3), $\Gamma\left(\theta_{T}\right) \neq \theta$. Hence, there exists $i$ between 1 and $n$ such that $\Gamma\left(\theta_{T}\right)(i) \neq \theta$. Now let $t \in T$. We have

$$
\theta_{T}\left(1_{G} ; i, i\right)=\left(\Psi\left(\theta_{T}\right)(i) ; \Gamma\left(\theta_{T}\right)(i), i\right)
$$

and

$$
\begin{aligned}
\theta_{T} t\left(1_{G} ; i, i\right) & =\theta_{T}(\Psi(t)(i) ; \Gamma(t)(i), i) \\
& =\left(\Psi\left(\theta_{T}\right)(\Gamma(t)(i)) \Psi(t)(i) ; \Gamma\left(\theta_{T}\right)(\Gamma(t)(i)), i\right) .
\end{aligned}
$$

Because $\theta_{T} t=\theta_{T}$ we obtain that $\Gamma\left(\theta_{T}\right)(\Gamma(t)(i))=\Gamma\left(\theta_{T}\right)(i)$. Now as $\Gamma\left(\theta_{T}\right)(i) \neq \theta, \Gamma(t)(i)=i$. Therefore, for every $t \in T$, we have $(i) \subseteq \Gamma(t)$. Because $\Gamma((g ; \alpha, \beta))=(\beta, \alpha, \theta)$ for every $(g ; \alpha, \beta) \in M$, it follows that $M \cap T=$ $\varnothing$. Let $M^{\prime}=\mathcal{M}^{0}\left(G,\{1, \ldots, i-1, i+1, \ldots, n\},\{1, \ldots, i-1, i+1, \ldots, n\} ; I_{n-1}\right)$. Since $(i) \subseteq \Gamma(t)$ and $\Gamma(t)$ restricted to $\{1, \ldots, n\} \backslash \Gamma(t)^{-1}(\theta)$ is injective for every $t \in T$, we get that $M^{\prime} T, T M^{\prime} \subseteq M^{\prime}$ and thus $M^{\prime} \cup T$ is a subsemigroup of $S$. Lemma 2.3 implies that there exist elements $w_{1}$ and $w_{2}$ of $T$ such that

$$
\begin{gathered}
(m, l) \subseteq \Gamma\left(w_{1}\right),(m)(l) \subseteq \Gamma\left(w_{2}\right) \\
\text { or }\left(\ldots, m, l, m^{\prime}, \ldots\right) \subseteq \Gamma\left(w_{1}\right),(l)\left(\ldots, m, m^{\prime}, \ldots\right) \subseteq \Gamma\left(w_{2}\right) \\
\text { or }\left[\ldots, k, m, \ldots, l, k^{\prime}, \ldots\right] \subseteq \Gamma\left(w_{1}\right),\left[\ldots, l, m, \ldots, k, k^{\prime}, \ldots\right] \subseteq \Gamma\left(w_{2}\right)
\end{gathered}
$$

for pairwise distinct numbers $l, m, m^{\prime}, k$ and $k^{\prime}$ between 1 and $n$. As $\Gamma\left(w_{1}\right)(o)$ $\neq o$ for $o \in\left\{l, m, m^{\prime}, k, k^{\prime}\right\}, i \notin\left\{l, m, m^{\prime}, k, k^{\prime}\right\}$. Hence $M^{\prime} \cup T$ is not nilpotent. As $T \cap M=\varnothing$ and $M \neq M^{\prime}, S \neq M^{\prime} \cup T$ and this is in contradiction with $S$ being minimal non-nilpotent.

The theorem shows that finite minimal non-nilpotent semigroups that are not a group belong to five classes. In order to get a complete classification, a remaining problem is to determine which semigroups in these classes are actually minimal non-nilpotent. In particular, one has to determine when precisely a union $M \cup T$ of an inverse semigroup $M=\mathcal{M}^{0}\left(G, n, n ; I_{n}\right)$ (with $G$ a nilpotent group) and a two-generated semigroup $T$ is minimal nonnilpotent. One might expect that the easiest case to deal with is when $M$ and $T$ are $\theta$-disjoint, i.e. the only possible joint element is the zero element $\theta$. In Corollary 4.2 we show that every finite minimal non-nilpotent semigroup which is of type $U_{3}, U_{4}$ or $U_{5}$ is an epimorphic image of such a semigroup. However, not every semigroup of type $U_{4}$ or $U_{5}$ that is a $\theta$-disjoint union of $M$ and $T$ is minimal non-nilpotent. Next we give examples of minimal non-nilpotent semigroups of type $U_{5}$ for which the maximal subgroups of 
$M$ are not trivial. We finish by constructing an infinite class of minimal non-nilpotent semigroups of type $U_{5}$ with $n \geq 5$ and $G$ the trivial group.

Note that in general the subsemigroups $T$ and $M$ of a minimal nonnilpotent semigroup $U_{m}$ (listed in Theorem 4.1) are not $\theta$-disjoint ( $\theta$-disjoint means that if there is a common element then it is $\theta$ ). We now show that $U_{m}$ (with $3 \leq n \leq 5$ ) is an epimorphic image of a semigroup built on $\theta$-disjoint semigroups.

Let $T$ be a semigroup with a zero $\theta_{T}$ and let $M$ be a nilpotent regular Rees matrix semigroup $\mathcal{M}^{0}\left(G, n, n ; I_{n}\right)$. Let $\Gamma$ be a representation of $T$ to the full transformation semigroup $\mathcal{T}_{\{1, \ldots, n\} \cup\{\theta\}}$ such that for every $t \in T, \Gamma(t)(\theta)=\theta$, $\left|\Gamma^{-1}(\theta)\right| \leq 1$ (as agreed before, by $\theta$ we also denote the constant map onto $\theta$ ), $\Gamma(t)$ restricted to $\{1, \ldots, n\} \backslash \Gamma(t)^{-1}(\theta)$ is injective and $\Gamma\left(\theta_{T}\right)=\theta$. Further, for every $t \in T$, let

$$
\Psi(t):\{1, \ldots, n\} \cup\{\theta\} \rightarrow G^{\theta}
$$

be a map (as considered in (2) ) such that $\Psi(t)(i) \neq \theta$ if and only if $\Gamma(t)(i) \neq \theta$ and $\Psi\left(t_{1} t_{2}\right)=\left(\Psi\left(t_{1}\right) \circ \Gamma\left(t_{2}\right)\right) \Psi\left(t_{2}\right)$ for every $t_{1}, t_{2} \in T$.

We define a semigroup denoted by

$$
S=\mathcal{M}^{0}\left(G, n, n ; I_{n}\right) \cup_{\Psi}^{\Gamma} T
$$

As sets this is the $\theta$-disjoint union of $\mathcal{M}^{0}\left(G, n, n ; I_{n}\right)$ and $T$ (i.e. the disjoint union with the zeros identified). The multiplication is such that $T$ and $M$ are subsemigroups,

$$
t(g ; i, j)= \begin{cases}(\Psi(t)(i) g ; \Gamma(t)(i), j) & \text { if } \Gamma(t)(i) \neq \theta \\ \theta & \text { otherwise }\end{cases}
$$

and

$$
(g ; i, j) t= \begin{cases}\left(g \Psi(t)\left(j^{\prime}\right) ; i, j^{\prime}\right) & \text { if } \Gamma(t)\left(j^{\prime}\right)=j \\ \theta & \text { otherwise }\end{cases}
$$

It can be easily verified that $S$ is associative.

Note that if $G=\{e\}$, then $\Psi(t)(i)=e$ if and only if $\Gamma(t)(i) \neq \theta$. In this case we denote $\Psi$ simply as $i d$.

It follows from Theorem 4.1 (and its proof) that the minimal non-nilpotent semigroup $\mathrm{S}$ of type $U_{m}$ (with $3 \leq n \leq 5$ ) is an epimorphic image of a semigroup of the type $\mathcal{M}^{0}\left(G, n, n ; I_{n}\right) \cup_{\Psi}^{\Gamma} T$, with $G$ a nilpotent group and $T$ a two-generated nilpotent semigroup with a zero.

Corollary 4.2. Every finite minimal non-nilpotent semigroup $S$ is an epimorphic image of one of the following semigroups:

(1) a Schmidt group,

(2) $U_{1}=\{e, f\}$ with $e^{2}=e, f^{2}=f$, ef $=f$ and $f e=e$,

(3) $U_{2}=\{e, f\}$ with $e^{2}=e, f^{2}=f$, ef $=e$ and $f e=f$,

(4) $\mathcal{M}^{0}\left(G, 2,2 ; I_{2}\right) \cup_{\Psi}^{\Gamma} T$ such that $T=\langle u\rangle \cup\{\theta\}$ with $\theta$ the zero of $S$, $u^{2^{k}}=1$ the identity of $T \backslash\{\theta\}$ (and of $S$ ) and $\Gamma(u)=(1,2)$.

(5) $\mathcal{M}^{0}\left(G, 3,3 ; I_{3}\right) \cup_{\Psi}^{\Gamma}\left\langle w_{1}, w_{2}\right\rangle$, with $\Gamma\left(w_{1}\right)=(2,1,3, \theta)$ and $\Gamma\left(w_{2}\right)=$ $(2,3, \theta)(1), w_{2} w_{1}^{2}=w_{1}^{2} w_{2}=w_{1}^{3}=w_{2} w_{1} w_{2}=\theta$. 
(6) $\mathcal{M}^{0}\left(G, n, n ; I_{n}\right) \cup_{\Psi}^{\Gamma}\left\langle v_{1}, v_{2}\right\rangle$, with

$$
\left[\ldots, k, m, \ldots, k^{\prime}, m^{\prime}, \ldots\right] \sqsubseteq \Gamma\left(v_{1}\right),\left[\ldots, k, m^{\prime}, \ldots, k^{\prime}, m, \ldots\right] \sqsubseteq \Gamma\left(v_{2}\right)
$$

for pairwise distinct numbers $k, k^{\prime}, m$ and $m^{\prime}$ between 1 and $n$, there do not exist distinct numbers $l_{1}$ and $l_{2}$ between 1 and $n$ such that $\left(l_{1}, l_{2}\right) \subseteq \Gamma(x)$ for some $x \in\left\langle v_{1}, v_{2}\right\rangle$ and there do not exist pairwise distinct numbers $o_{1}, o_{2}$ and $o_{3}$ between 1 and $n$ such that $\left(o_{2}, o_{1}, o_{3}, \theta\right)$ $\subseteq \Gamma\left(y_{1}\right),\left(o_{2}, o_{3}, \theta\right)\left(o_{1}\right) \subseteq \Gamma\left(y_{2}\right)$ for some $y_{1}, y_{2} \in\left\langle v_{1}, v_{2}\right\rangle$.

An example of a semigroup of type $U_{4}$ that is not minimal non-nilpotent. Consider the following semigroup

$$
S=\mathcal{M}^{0}\left(\left\{1_{G}, g\right\}, 3,3 ; I_{3}\right) \cup_{i d}^{\Gamma}\langle w, v\rangle
$$

with $v^{2}=v^{3}, w v^{2}=w v, v w=v^{2} w, w^{2}=w v w=w v^{2} w, v w^{2}=w^{2} v=w^{3}=$ $v w v=\theta, \Gamma(w)=(2,1,3, \theta)$ and $\Gamma(v)=(2,3, \theta)(1)$. Clearly, $\mathcal{M}^{0}\left(\left\{1_{G}\right\}, 3,3 ; I_{3}\right.$ )$\cup_{i d}^{\Gamma}\langle w, v\rangle$ is a proper semigroup. The latter is minimal non-nilpotent and thus $S$ is not minimal non-nilpotent.

An example of a finite minimal non-nilpotent semigroup of type $U_{5}$ with trivial maximal subgroups in $M$.

Let

$$
S=\mathcal{M}^{0}\left(G, 4,4 ; I_{4}\right) \cup_{\Psi}^{\Gamma}\langle w, v\rangle
$$

with $G=\left\{1_{G}, g\right\}$ a cyclic group of order $2, w^{2}=v^{2}=w v=v w=\theta$,

$$
\begin{gathered}
\Gamma(w)=(4,1, \theta)(3,2, \theta) \text { and } \Gamma(v)=(4,2, \theta)(3,1, \theta), \\
\Psi(w)(4)=\Psi(w)(3)=\Psi(v)(4)=1, \quad \Psi(v)(3)=g \text { and }\langle w, v\rangle=\{w, v, \theta\} .
\end{gathered}
$$

Since

$$
\Gamma\left(\left(1_{G} ; 3,1\right)\right)=\lambda_{2}\left(\Gamma\left(\left(1_{G} ; 3,1\right)\right), \Gamma\left(\left(1_{G} ; 4,2\right)\right), \Gamma(w), \Gamma(v)\right)
$$

and

$$
\Gamma\left(\left(1_{G} ; 4,2\right)\right)=\rho_{2}\left(\Gamma\left(\left(1_{G} ; 3,1\right)\right), \Gamma\left(\left(1_{G} ; 4,2\right)\right), \Gamma(w), \Gamma(v)\right),
$$

we obtain that $S$ is not nilpotent. Suppose that a subsemigroup $S^{\prime}$ of $S$ is not nilpotent. By Lemma 2.1, there exists a positive integer $m$, distinct elements $x, y \in S^{\prime}$ and elements $w_{1}, w_{2}, \ldots, w_{m} \in S^{\prime 1}$ such that $x=$ $\lambda_{m}\left(x, y, w_{1}, w_{2}, \ldots, w_{m}\right)$ and $y=\rho_{m}\left(x, y, w_{1}, w_{2}, \ldots, w_{m}\right)$. As $\langle w, v\rangle$ is nilpotent,

$$
\left\{x, y, w_{1}, w_{2}, \ldots, w_{m}\right\} \cap \mathcal{M}^{0}\left(G, 4,4 ; I_{4}\right) \neq \varnothing
$$

and because $\mathcal{M}^{0}\left(G, 4,4 ; I_{4}\right)$ is an ideal of $S, x$ and $y$ are nonzero elements in $\mathcal{M}^{0}\left(G, 4,4 ; I_{4}\right)$. Since $S^{\prime}$ is not nilpotent and $\mathcal{M}^{0}\left(G, 4,4 ; I_{4}\right)$ is nilpotent, at least one element of the set $\left\{w_{1}, \ldots, w_{m}\right\}$ is not in $\mathcal{M}^{0}\left(G, 4,4 ; I_{4}\right)$. As before, without loss of generality, we may suppose that $w_{1} \notin \mathcal{M}^{0}\left(G, 4,4 ; I_{4}\right)$. Write $x=\left(g_{1} ; n_{1}, n_{2}\right)$ and $y=\left(g_{2} ; n_{3}, n_{4}\right)$, for some $1 \leq n_{1}, n_{2}, n_{3}, n_{4} \leq 4$ and $g_{1}, g_{2} \in G$.

If $w_{1}=w$ then $\left\{\left(n_{1}, n_{2}\right),\left(n_{3}, n_{4}\right)\right\}=\{(3,1),(4,2)\}$ and it can be easily verified that $w_{2}=v$. 
It also is easily verified that $\mathcal{M}^{0}\left(\left\{1_{G}\right\}, 4,4 ; I_{4}\right)$ is a subsemigroup of

$$
\left\langle\Gamma\left(\left(g_{1} ; 3,1\right)\right), \Gamma\left(\left(g_{2} ; 4,2\right)\right), \Gamma(w), \Gamma(v)\right\rangle .
$$

So, $\mathcal{M}^{0}\left(\left\{1_{G}\right\}, 4,4 ; I_{4}\right) \subseteq \Gamma\left(S^{\prime}\right)$. Therefore, for every pair $1 \leq \alpha, \beta \leq 4$, there exists an element $p \in S^{\prime}$ such that $\Gamma(p)=(\beta, \alpha, \theta)$. As $\Gamma(w)=(4,1, \theta)(3,2, \theta)$ and $\Gamma(v)=(4,2, \theta)(3,1, \theta)$ and $\langle w, v\rangle=\{w, v, \theta\}$, we obtain that there exists an element $h \in\left\{1_{G}, g\right\}$ such that $p=(h ; \alpha, \beta)$.

If $S \neq S^{\prime}$ then there exists an element $(k ; i, j) \in \mathcal{M}^{0}\left(G, 4,4 ; I_{4}\right)$ such that $(k ; i, j) \notin S^{\prime}$. Now suppose that $\left(n_{1}, n_{2}\right)=(3,1)$ and $\left(n_{3}, n_{4}\right)=(4,2)$. Then

$$
\begin{gathered}
v\left(g_{1} ; 3,1\right) v=\left(g g_{1} g ; 1,3\right), \quad w\left(g_{2} ; 4,2\right) w=\left(g_{2} ; 1,3\right), \\
v\left(g_{1} ; 3,1\right) w=\left(g g_{1} ; 1,4\right), \quad w\left(g_{2} ; 4,2\right) v=\left(g_{2} ; 1,4\right) .
\end{gathered}
$$

Since $g_{1}, g_{2} \in\left\{1_{G}, g\right\}$ we get that both $\left(1_{G} ; 1,3\right)$ and $(g ; 1,3)$, or both $\left(1_{G} ; 1,4\right)$ and $(g ; 1,4)$ are in $S^{\prime}$. Suppose that both $\left(1_{G} ; 1,3\right)$ and $(g ; 1,3)$ are in $S^{\prime}$. As proved above, there exist elements $k_{1}, k_{2} \in G$ such that $\left(k_{1} ; i, 1\right),\left(k_{2} ; 3, j\right) \in S^{\prime}$. Then $\left(k_{1} ; i, 1\right)\left(1_{G} ; 1,3\right)\left(k_{2} ; 3, j\right),\left(k_{1} ; i, 1\right)(g ; 1,3)\left(k_{2}\right.$; $3, j) \in S^{\prime}$ and thus $\left(k_{1} k_{2} ; i, j\right),\left(k_{1} g k_{2} ; i, j\right) \in S^{\prime}$. Since $k, k_{1}, k_{2} \in\left\{1_{G}, g\right\}$ we get that $(k ; i, j)$ is in $S^{\prime}$, a contradiction. So, $S=S^{\prime}$ in this case.

Similarly, $\left(1_{G} ; 1,4\right),(g ; 1,4) \in S^{\prime}$ leads to $S=S^{\prime}$. Hence, we have proved that $S=S^{\prime}$ if $w_{1}=w$. If $w_{1}=v$, then one proves in an analogous manner that $S=S^{\prime}$. So, it follows that $S$ is a minimal non-nilpotent semigroup of type $U_{5}$.

An infinite class of finite minimal non-nilpotent semigroups of type $U_{5}$. Let $n \geq 5$ and consider $\mathcal{M}^{0}\left(\{e\}, n, n ; I_{n}\right)$ as a subsemigroup of the full transformation semigroup (see (3) $)$ on $\{1, \ldots, n\} \cup\{\theta\}$, i.e. we identify $(e ; i, j)$ with the cycle $(j, i, \theta)$ if $i \neq j$ and $(e ; i, i)$ with the permutation $(i)$. Let

$$
Y_{n}=\mathcal{M}^{0}\left(\{e\}, n, n ; I_{n}\right) \cup_{i d}^{\Gamma}\langle w, v\rangle
$$

and

$$
\Gamma(w)=(2,3, \theta)(4,1, \theta), \Gamma(v)=(2,1, \theta)(n, n-1, \ldots, 5,4,3, \theta) .
$$

It can be easily verified that

$$
\begin{gathered}
\Gamma\left(v^{p} w^{q}\right)=\Gamma\left(w^{k}\right)=\Gamma\left(v^{l}\right)=\theta \text { for } p, q \geq 1, k \geq 2, l \geq n-2, \\
\Gamma\left(w^{q} v^{p}\right)=\theta \text { for } q \geq 2, p \geq 1, \\
\Gamma\left(w v^{p}\right)=(p+4,1, \theta) \text { for } n-4 \geq p \geq 1, \Gamma\left(w v^{p}\right)=\theta \text { for } p>n-4 \text { and } \\
\Gamma\left(a w v^{p}\right)=\theta \text { for } p \geq 1, a \in\langle w, v\rangle .
\end{gathered}
$$

Because $\left|\Gamma^{-1}(\theta)\right|=1$ we thus obtain that $v^{p} w^{q}=w^{k}=v^{l}=\theta$ for $p, q \geq 1, k \geq$ $2, l \geq n-2, w^{q} v^{p}=\theta$ for $q \geq 2, p \geq 1$ and $a w v^{p}=\theta$ for $p \geq 1, a \in\langle w, v\rangle$. So,

$$
\langle w, v\rangle=\left\{w, v, \ldots, v^{n-3}, w v, \ldots, w v^{n-4}, \theta\right\}
$$

and clearly $\langle w, v\rangle^{n}=\{\theta\}$. Therefore $\langle w, v\rangle$ is nilpotent.

We claim that $Y_{n}$ is minimal non-nilpotent. To prove this, suppose that $Y$ is a subsemigroup of $Y_{n}$ that is not nilpotent. We need to prove that $Y=Y_{n}$. As before, there exists a positive integer $m$, distinct elements $x, y \in \mathcal{M}^{0}\left(\{e\}, n, n ; I_{n}\right)$ and elements $w_{1}, w_{2}, \ldots, w_{m} \in Y^{1}$ with $w_{1} \notin$ 
$\mathcal{M}^{0}\left(\{e\}, n, n ; I_{n}\right)$ such that $x=\lambda_{m}\left(x, y, w_{1}, w_{2}, \ldots, w_{m}\right), y=\rho_{m}\left(x, y, w_{1}, w_{2}\right.$ $\left., \ldots, w_{m}\right)$. Write $x=\left(e ; n_{1}, n_{2}\right), y=\left(e ; n_{3}, n_{4}\right)$ for some $1 \leq n_{1}, n_{2}, n_{3}, n_{4} \leq n$. Since $x \neq y,\left(n_{1}, n_{2}\right) \neq\left(n_{3}, n_{4}\right)$.

Since $\Gamma\left(x w_{1} y\right)$ and $\Gamma\left(y w_{1} x\right)$ are nonzero, $\Gamma\left(w_{1}\right)\left(n_{3}\right)=n_{2}$ and $\Gamma\left(w_{1}\right)\left(n_{1}\right)=$ $n_{4}$. Now as $\Gamma\left(w v^{p}\right)=(p+4,1, \theta)$ for $n-4 \geq p \geq 1$ and $\left(n_{1}, n_{2}\right) \neq\left(n_{3}, n_{4}\right)$, $w_{1} \notin\left\{w v, \ldots, w v^{n-4}\right\}$. Similarly $w_{2} \notin\left\{w v, \ldots, w v^{n-4}\right\}$. So, $w_{1}=w$ or $w_{1}=v^{k}$ for some $n-3 \geq k \geq 1$.

Suppose that $w_{1}=v^{k}$ for some $1 \leq k \leq n-3$. Since $\Gamma\left(x w_{1} y\right)$ and $\Gamma\left(y w_{1} x\right)$ are nonzero, $\Gamma\left(v^{k}\right)\left(n_{3}\right)=n_{2}$ and $\Gamma\left(v^{k}\right)\left(n_{1}\right)=n_{4}$. Hence $\lambda_{1}=\left(e ; n_{1}, n_{4}\right), \rho_{1}=$ $\left(e ; n_{3}, n_{2}\right)$. If $w_{2}=w$ then $\Gamma(w)\left(n_{1}\right)=n_{2}$ and $\Gamma(w)\left(n_{3}\right)=n_{4}$. Since $\left(n_{1}, n_{2}\right) \neq\left(n_{3}, n_{4}\right)$ and $\Gamma(w)=(2,3, \theta)(4,1, \theta), n_{1}=4, n_{2}=1, n_{3}=2, n_{4}=3$ or $n_{1}=2, n_{2}=3, n_{3}=4, n_{4}=1$. Now as $\Gamma\left(v^{k}\right)\left(n_{3}\right)=n_{2}, \Gamma\left(v^{k}\right)\left(n_{1}\right)=n_{4}$ and $\Gamma\left(v^{k}\right)(2)=\theta$ for $k>1, v^{k}=v$ and thus $Y=Y_{n}$. So we may assume that there exists $1 \leq l \leq n$ such that $w_{2}=v^{l}$. Similarly we have $\Gamma\left(v^{l}\right)\left(n_{1}\right)=n_{2}$ and $\Gamma\left(v^{l}\right)\left(n_{3}\right)=n_{4}$. It can be easily verified that $n_{4}=n_{1}-k=n_{3}-l, n_{2}=$ $n_{1}-l=n_{3}-k$. Then $k-l=l-k$ and thus $k=l$. Hence $n_{4}=n_{2}, n_{1}=n_{3}$, a contradiction.

Finally suppose that $w_{1}=w$. As $\Gamma\left(w_{1}\right)\left(n_{3}\right)=n_{2}, \Gamma\left(w_{1}\right)\left(n_{1}\right)=n_{4}, \Gamma(w)=$ $(2,3, \theta)(4,1, \theta)$ and $\left(n_{1}, n_{2}\right) \neq\left(n_{3}, n_{4}\right),\{x, y\}=\{(e ; 4,3),(e ; 2,1)\}$ and thus $\rho_{1}, \lambda_{1} \in\{(e ; 2,3),(e ; 4,1)\}$. Now as $\Gamma\left(\rho_{1} w_{2} \lambda_{1}\right)$ and $\Gamma\left(\lambda_{1} w_{2} \rho_{1}\right)$ are nonzero, it follows that $(2,1, \theta) \subseteq \Gamma\left(w_{2}\right),(4,3, \theta) \subseteq \Gamma\left(w_{2}\right)$. Since $\Gamma\left(v^{k}\right)(2)=\theta$ for $k>1$ and $\Gamma(w)(2)=3$, one then obtains that $w_{2}=v$. Therefore $Y=Y_{n}$. It follows that indeed $Y_{n}$ is minimal non-nilpotent.

\section{REFERENCES}

[1] A. Abdollahi and M. Zarrin, Non-nilpotent graph of a group, Comm. Algebra 38 (12) (2010), 4390-4403.

[2] A.H. Clifford and G.B. Preston, The Algebraic Theory of Semigroups, Vol. I. Mathematical Surveys, No. 7 American Mathematical Soc., Providence, R.I., 1967.

[3] E. Jespers and J. Okninski, Nilpotent semigroups and semigroup algebras, J. Algebra 169 (3) (1994), 984-1011.

[4] E. Jespers and J. Okninski, Semigroup algebras and maximal orders, Canadian Math. Bulletin 42 (3) (1999), 298-306.

[5] E. Jespers and J. Okninski, Noetherian semigroup algebras, Algebras and Applications 7, Springer, Dordrecht, 2007.

[6] E. Jespers and D. Riley, Nilpotent linear semigroups, International Journal of Algebra and Computation 16 (1) (2006), 141-160.

[7] E. Jespers and M.H. Shahzamanian, A description of a class of finite semigroups that are near to being Malcev nilpotent, J. Algebra Appl. 12, 1250221 (2013) [26 pages] DOI: $10.1142 /$ S0219498812502210.

[8] E. Jespers and M.H. Shahzamanian, The non-nilpotent graph of a semigroup, Semigroup Forum 85 (1) (2012), 37-57.

[9] G. Lallement, On nilpotency in semigroups, Pacific J. Math. 42 (1972), 693-700.

[10] H. Lausch and W. Nobauer, Algebra of Polynomials, North-Holland Mathematical Library, Vol. 5. North-Holland Publishing Co., Amsterdam-London; American Elsevier Publishing Co., Inc., New York, 1973.

[11] A.I. Malcev, Nilpotent semigroups, Uc. Zap. Ivanovsk. Ped. Inst. 4 (1953), 107-111 (in Russian). 
[12] B.H. Neumann, Tekla Taylor, Subsemigroups of nilpotent groups, Proc. Roy. Soc, Ser.A 274 (1963), 1-4.

[13] J. Okninski, Nilpotent semigroup of matrices, Math. Proc. Camb. Phil. Soc. 120(4) 11 (1996), 617-630.

[14] J. Okninski, Semigroup Algebras, Monographs and Textbooks in Pure and Applied Mathematics, 138. Marcel Dekker, Inc., New York, 1991.

[15] O.Y. Schmidt, Groups whose all subgroups are special, Mat.Sb., 31 (1924), 366-372 (In Russian).

[16] W.R. Scott, Group Theory, Second edition. Dover Publications, Inc., New York, 1987.

Eric Jespers and M.H. Shahzamanian, Department of Mathematics, VriJe Universiteit Brussel, Pleinlaan 2, 1050 Brussel, Belgium

E-mail address: efjesper@vub.ac.be, m.h.shahzamanian@vub.ac.be

Current address M.H. Shahzamanian, Centro de Matematica Universidade do Porto, Rua do Campo Alegre 687, 4169-007 Porto, Portugal

E-mail address: m.h.shahzamanian@fc.up.pt 